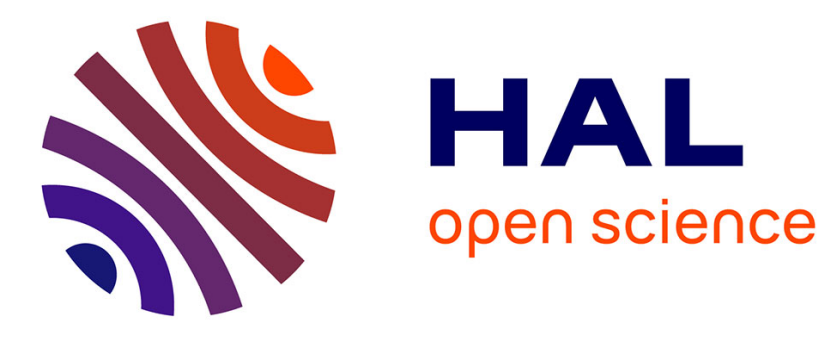

\title{
Topology on digital label images
}

Loïc Mazo, Nicolas Passat, Michel Couprie, Christian Ronse

\section{To cite this version:}

Loïc Mazo, Nicolas Passat, Michel Couprie, Christian Ronse. Topology on digital label images. Journal of Mathematical Imaging and Vision, 2012, 44 (3), pp.254-281. 10.1007/s10851-011-0325-8 . hal00727353

\section{HAL Id: hal-00727353 \\ https://hal.science/hal-00727353}

Submitted on 26 Feb 2018

HAL is a multi-disciplinary open access archive for the deposit and dissemination of scientific research documents, whether they are published or not. The documents may come from teaching and research institutions in France or abroad, or from public or private research centers.
L'archive ouverte pluridisciplinaire HAL, est destinée au dépôt et à la diffusion de documents scientifiques de niveau recherche, publiés ou non, émanant des établissements d'enseignement et de recherche français ou étrangers, des laboratoires publics ou privés. 


\section{Journal of Mathematical Imaging and Vision \\ Topology on digital label images \\ --Manuscript Draft--}

\begin{tabular}{|c|c|}
\hline Manuscript Number: & JMIV-806R2 \\
\hline Keywords: & digital imaging; topology; label images; homotopy; simple points. \\
\hline Corresponding Author: & $\begin{array}{l}\text { Loïc Mazo } \\
\text { Illkirch, FRANCE }\end{array}$ \\
\hline \multicolumn{2}{|c|}{$\begin{array}{l}\text { Corresponding Author Secondary } \\
\text { Information: }\end{array}$} \\
\hline \multicolumn{2}{|c|}{ Corresponding Author's Institution: } \\
\hline \multicolumn{2}{|c|}{ First Author Secondary Information: } \\
\hline \multirow[t]{4}{*}{ All Authors: } & Loïc Mazo \\
\hline & Nicolas Passat \\
\hline & Michel Couprie \\
\hline & Christian Ronse \\
\hline All Authors Secondary I & \\
\hline
\end{tabular}




\title{
Topology on digital label images JMIV-806 Answers to Editor / Reviewers
}

\author{
Loïc Mazo, Nicolas Passat, Michel Couprie, Christian Ronse
}

December 19, 2011

\section{Response to the Editor:}

Editor: We have received the reports from our advisors on your manuscript, "Topology on digital label images", which you submitted to Journal of Mathematical Imaging and Vision.

Based on the advice received, the Editor feels that your manuscript could be accepted for publication should you be prepared to incorporate minor revisions. When preparing your revised manuscript, you are asked to carefully consider the reviewer comments, which are attached, and submit a list of responses to the comments. Your list of responses should be uploaded as a file in addition to your revised manuscript.

Authors: We would like to thank the Editor for the management of the review process of this manuscript. It has been revised by taking into account the suggestions of the reviewer.

\section{Response to Reviewer \#2:}

Authors: We would like to thank the Reviewer for having taken time to evaluate our manuscript. A point-by-point response to each of the issues raised by the Reviewer is given below.

Referee: The authors have made different corrections that significantly improve their article. However, I would greatly appreciate that some efforts will be made to give an easier access to the paper content for novice readers.

For example, add some references at the beginning of section 2.1, the books of Munkres (Elements of Algebraic Topology), Hatcher (Algebraic Topology) or Giblin (Graphs, Surfaces and Homology) are valuable references for novice reader. When adding references pay particular attention to the availability of it and be sure that the style and the way of the concepts are presented are adapted to the "modern" readers. For example, page 4 line 36: of course Whitehead as defined elementary transformations on complexes but I am not that this reference is easy to find and it would really help the reader, again a book like Giblin's book is more adapted. 
Authors: We have added these references together with Maunder (Algebraic Topology) and May (A Concise Course in Algebraic Topology).

Page 4 line 36: we have given two references, the original one (which is freely available on the web) and Giblin's book.

Referee: Page 4, line 9, second column: You talk about topological spaces with base points without introducing them. After lines 12 and following: The tentative explanation about weak homotopy equivalence is very hard to follow if you don't know what is the homotopy class of a map.

Authors: A subsection about homotopy have been added in order to introduce the minimal knowledge of algebraic topology that is needed in the paper.

Referee: page 5 line 2: exemple 24 is very far from this text.

Authors: A new figure has been added just after the text.

Referee: page 5 section 2.3: in the first paragraphe you talk about A-space and the about Alexandroff space this is puzzling.

Authors: This has been corrected.

Referee: page 6 , lines $45-50$. You use property 3 that is given below and you refer to property 6 that is on page 6 in section 2.4 after the introduction of unipolar points. Please reconsider the redaction of this paragraph.

Authors: The paragraph has been rewritten (and the references to properties 3 and 6 have been removed). 
1

\title{
Topology on digital label images
}

\author{
Loïc Mazo · Nicolas Passat - Michel Couprie · Christian Ronse
}

Received: date / Accepted: date

Abstract In digital imaging, after several decades devoted to the study of topological properties of binary images, there is an increasing need of new methods enabling to take into (topological) consideration $n$-ary images (also called label images). Indeed, while binary images enable to handle one object of interest, label images authorise to simultaneously deal with a plurality of objects, which is a frequent requirement in several application fields. In this context, one of the main purposes is to propose topology-preserving transformation procedures for such label images, thus extending the ones (e.g., growing, reduction, skeletonisation) existing for binary images. In this article, we propose, for a wide range of digital images, a new approach that permits to locally modify a label image, while preserving not only the topology of each label set, but also the topology of any arrangement of the labels understood as the topology of any union of label sets. This approach enables in particular to unify and extend some previous attempts devoted to the same purpose.

Keywords digital imaging $\cdot$ topology $\cdot$ label images · homotopy $\cdot$ simple points

The research leading to these results has received funding from the French Agence Nationale de la Recherche (Grant Agreement ANR2010-BLAN-0205).

Loïc Mazo, Nicolas Passat, Christian Ronse

Université de Strasbourg, LSIIT, UMR CNRS 7005, France

Tel.: +33-368854413

Fax: $+33-368854455$

E-mail: loic.mazo@unistra.fr

Loïc Mazo, Michel Couprie

Université Paris-Est, Laboratoire d'Informatique Gaspard-Monge, Équipe A3SI, ESIEE Paris, France

\section{Introduction}

In a digital image, when performing processes such as registration, deformation or thinning, the preservation of the topological properties of the objects contained in the image (e.g., connected components, tunnels, cavities, etc.) is an important requirement. For 50 years, several tools enabling the analysis (adjacency graphs, digital fundamental groups, homology groups -see, e.g., [1,2,3]) and the modification under topological constraints (simple points, P-simple points, simple sets -see, e.g., [4,5,6,7]) of binary images have been proposed and used. Nevertheless, in many fields (e.g., medical imaging, remote sensing, computer vision), an image is generally composed of several objects, and it is often important to understand or maintain their topological properties all together, that is the topology of each and the topology of the scene. In such images, the objects are characterised by specific labels on which there generally exists no meaningful order relation (unlike grey-level images for instance).

\subsection{Previous works}

To the best of our knowledge, the literature about topology in label images is quite limited and generally motivated by practical considerations. The most common approach is to consider only one label at a time, the other labels being momentarily considered as a part of the background. However, except in the most simple cases where the label configuration leads to a binary modelling (see, e.g., [8,9]), one cannot directly deal with the relations between the labels but only with the topology of each label and of its associated background [10,11,12] (if necessary, one uses in addition an adjacency tree between labels in order to control their topological relations). These methods are often used with a cost function, which depends on the applicative context, whose 


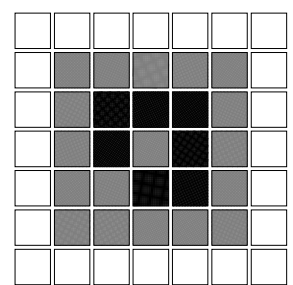

Fig. 1 An image with two labels (in grey and black). If we consider the grey label as the object of the picture using the $(8,4)$-adjacency pair (8-adjacency for the object and 4-adjacency for the background), the object is a ring. The black pixels together then form the inner component of the background, while the white pixels form the outer component. However, if we now consider the black pixels as the object (still in 8-adjacency), rejecting grey pixels to the background, these latters must be understood with the 4-adjacency and they appear to have two connected components, one inside the black torus and one outside.

purpose is to assign a given label, or not, to a point of the image. Thereby points go from background to a label or vice versa but not from a label to another. Note that some points may sometimes take an undetermined status since they cannot be assigned a label without breaking a topology defined by an a priori knowledge or to avoid object crossings when the objects are seen under the filter of the 8-adjacency in the plane or 26-adjacency in the space (see Figure 11. The question of the adjacencies to be used in a digital label image is a recurrent issue. Indeed, in digital topology, in the framework developed by Rosenfeld [13], the object and the background of an image are understood with different (dual) adjacencies [14]. So, when objects in a label image are processed one at a time, being alternately the object and part of the background, they are inevitably seen under two distinct adjacencies 1 . For instance, an object can have one connected component at one step of the process and two components at the next step though no change did occur on the image (see Figure 1).

To overcome this problem, a class of "well composed" images has been defined in which the same adjacency relation can be used for the object and the background. This adjacency relation is necessarily the 4-adjacency in $2 \mathrm{D}$ images and the 6-adjacency in 3D images [17]. This class of images is obtained by excluding all the images in which at least one of the three configurations depicted on Figure 2 appears. In other words, it is assumed in these images that the boundaries of the objects (viewed as an union of $n$-cubes) are $(n-1)$-manifolds. In the case where label images present forbidden configurations, an algorithm has been proposed to

1 This problem is sometimes disregarded. For instance, in [15] (proof of Proposition 2), it is claimed "Since the 18-neighbourhood of $x$ is limited to binary case, and by definition of simple points the topology of the complementary of $R$ is preserved: we can deduce that the topology of $X$ [the complementary of $R$ in the 18-neighbourhood of $x]$ is also preserved, and thus that $x$ is simple for $X$ ". It is not clear here what is meant by preserving topology. However, in the framework of simple points [16], it is not true in general that we can swap the object and the background without swapping together the adjacency pair.

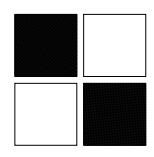

(a)

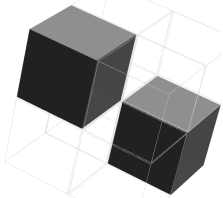

(b)

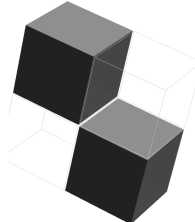

(c)
Fig. 2 Forbidden configurations in (binary) well composed images. (a) In $\mathbb{Z}^{2}$. (b,c) In $\mathbb{Z}^{3}$ (configuration (b) shall not appear neither in the object nor in the background). A label image is well composed if each binary image obtained by isolating a particular label is well composed.

dispose of them [18]. However, since the objects identified by the labels are sequentially "repaired", one needs first to determine an order on the labels, and this order biases the result.

Another approach [19] takes further the specificity of label images into account. A notion of "homotopy set" is defined, which is the set of the labels that can be assigned to a point without modification on the topology of each label and of its complement in the image. A local criterion is provided to decide whether a particular label belongs to the homotopy set of a point or not. Thereby, a point can move from a label to another and not solely from the background to a label or vice versa.

In [20], the authors go further and require, before any change of label at a point, the guarantee that not only the topology of each label will be preserved but also the topology of the unions of two labels in 2D images and of three labels in 3D images (see Figure 3). Nevertheless, this request is not sufficient. Figure 3 (c) provides a counterexample in $2 \mathrm{D}$ where there is the need to consider the union of three labels.

In [15], the authors study 3D label images with a frontier approach. The 3D image is divided into regions which are 6connected (hence, the configurations of Figure 2 cannot occur) and in which the voxels share the same label. Moreover, they only take into account the 6-adjacency between regions. To move a voxel $x$ from a region $A$ to another region $B$, the authors make requirements on surfaces between $x$ and $A \backslash\{x\}$ (resp. between $x$ and $B \backslash\{x\}$ ): they have to be homeomorphic to a 2-disk. Furthermore, for each region $C 6$-adjacent to $x$, the frontier between the regions $A$ and $C$ before the move (resp. between $B$ and $C$ after the move), must collapse 2 onto the corresponding frontier after the move (resp. before the move).

\footnotetext{
${ }^{2}$ Here, collapse is the classical operation on complexes defined by Whitehead [21] (see Section 2.2).
} 


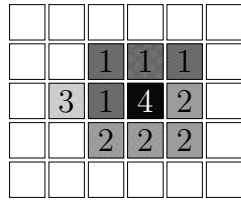

(a)

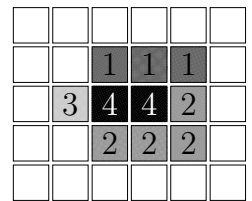

(b)

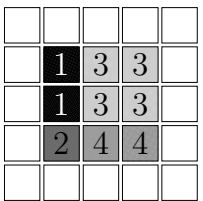

(c)
Fig. 3 (a) An image with four labels. (b) The label of a single pixel has changed. Neither the topologies of the labels nor of their complements in the image are modified. However, the topology of the partition is not preserved in the sense that the union $1+2$ becomes contractible, $1+3$ is split into two components in 4-adjacency, $3+4$ loses a component, $2+3+4$ loses a component in 4-adjacency. (c) This example is from [20]. The authors observe that, if we look at the picture with the $(8,4)$ adjacency pair, the central pixel can move from 3 to 2 without altering the topologies of the four labels and of the six pairs of labels but they do not take into consideration the union $1+3+4$ though it passes from a ball to a ring. Observe also that the well-composedness of this image is destroyed by the move of the central pixel from 3 to 2 .

\subsection{Purpose}

The aim of this article is to study the topology of label images, following the idea to preserve any union of labels, which amounts to require topologically sound procedures on digital label images not to change the topological characteristics of the sets of a partition of $\mathbb{Z}^{n}$ and of any coarser partition of the initial one. In other words, one could say that the actual set of objects in a digital label image is the power set of the partition. We have adopted a theoretical standpoint with the will to cover a wide range of situations. In our framework, we do not make any assumption on the topologies of individual objects (we do not use a priori knowledge) and there is no forbidden configurations. Weak homotopy equivalence in finite spaces (which corresponds to homotopy equivalence in continuous ones) is used to perform topological comparisons. To avoid the pitfall of distinct adjacency pairs on the same object described above, we embed the digital space of the image into a richer space equipped with a genuine topology, that is a poset whose minimal points are the points of the digital image. This enrichment of the space leads us to embed also the label set into a richer one, namely an atomistic lattice whose atoms are the labels of the digital image. Thereby, we can extend the digital image on its poset, assigning extended labels to new points, and we can define gradual modifications of the images more adapted to topology preservation.

\subsection{Contribution and structure of the article}

The remainder of this article is organised as follows.

Section 2 gathers results on binary images on which relies our work. It is intended to make the article self-contained and to introduce our notations. The last subsection of Sec- tion2 establishes, in particular, two new results whose proofs are provided in Appendix Band $\mathrm{C}$

In Section 3, we introduce our framework for the topological understanding of label images. We describe a first tool to locally modify such a label image while keeping unchanged all homotopy groups of the objects and their unions (to be more precise, we have weak homotopy equivalences). When the poset is the space $\mathbb{F}^{n}$ of cubical complexes defined in Section 2, our tool keeps also unchanged the homotopy groups of the complements. Furthermore, the changes can be processed in parallel under certain conditions, thus leading to well-balanced algorithms.

In Section 4, we are interested in images in which the sets of points that share a label (we say the support of the label) are closed sets, as in $(26,6)$ digital images. In this case, we define an elementary modification, named $c u t$, inspired by collapses. It has the same (good) topological properties as the one defined in Section 3 while the supports of the labels remain closed sets.

In Section 5, we study regular images in which the label of a point in the poset is defined by the labels of the minimal points beneath it. Regular images can be built from digital images defined on $\mathbb{Z}^{n}$ and we have proved in [22] that, when the poset is the space of cubical complexes, this construction puts in one-to-one correspondence the connected components of the regular image with the ones of the digital image. Moreover, it induces isomorphisms between the fundamental groups of the regular image and the digital fundamental groups of the digital image (as defined in [2]). In regular images, we give conditions for cuts to preserve regularity allowing thereby to modify a regular image in a topologically sound manner, the result being also a regular image (allowing to go back to $\mathbb{Z}^{n}$ ).

Section [ concludes this paper and describes further works in preparation.

\section{Simplicity in sets}

The aim of this section is to gather notions and results on which relies this work, and also to present our notations. Note that in Section 2.8, we establish (new) results which are specific to complexes. Operations and relations on functions (in particular, on images) will always be implicitly pointwise ones.

\subsection{Homotopy}

Two continuous maps $f, g: X \rightarrow Y$ are homotopic if there exists a continuous map, called a homotopy, $h: X \times[0,1] \rightarrow$ $Y$ such that $h(x, 0)=f(x)$ and $h(x, 1)=g(x)$ for all $x \in X$. The spaces $X$ and $Y$ are homotopy equivalent (or have the same homotopy type) if there exist two continuous maps 
$f: X \rightarrow Y$ and $g: Y \rightarrow X$, called homotopy equivalences, such that $g \circ f$ is homotopic to the identity map $\operatorname{id}_{X}$ and $f \circ g$ is homotopic to $\operatorname{id}_{Y}$. If $X$ and $Y$ are homeomorphic, they are homotopy equivalent: given a homeomorphism $\varphi$ between $X$ and $Y, \varphi$ and $\varphi^{-1}$ are homotopy equivalences between $X$ and $Y$. The converse is not true in general (for example, a ball is homotopy equivalent - but not homeomorphic- to a point). A topological space is contractible if it has the homotopy type of a single point. Let $X$ be a topological space. Two paths $p, q$ in $X$ are equivalent if they have the same extremities (i.e., $p(0)=q(0)$ and $p(1)=q(1)$ ) and are homotopic by an homotopy $h$ such that $h(0, u)=p(0)=q(0)$ and $h(1, u)=p(1)=q(1)$ for all $u \in[0,1]$. It is easy to check that this relation on paths is actually an equivalence relation. We write $[p]$ for the equivalence class of $p$. If $p, q$ are two paths in $X$ such that $p(1)=q(0)$ we can define the product $p \cdot q$ by:

$(p \cdot q)(t)= \begin{cases}p(2 t) & \text { if } t \in\left[0, \frac{1}{2}\right] \\ q(2 t-1) & \text { if } t \in\left[\frac{1}{2}, 1\right] .\end{cases}$

This product is well defined on equivalence classes by $[p]$. $[q]=[p \cdot q]$. Let $x$ be a point of $X$. A loop at $x$ is a path in $X$ which starts and ends at $x$. The product of two loops at $x$ is a loop at $x$ and the set $\pi_{1}(X, x)$ of equivalence classes of loops at $x$ is a group for this product. It is called the fundamental group of $X$ (with basepoint $x$ ) or the first homotopy group of $X$. If $X$ is path-connected, the group $\pi_{1}(X, x)$ does not depend on the basepoint (i.e., for any points $x, y \in X, \pi_{1}(X, x)$ and $\pi_{1}(X, y)$ are isomorphic). Higher homotopy groups, denoted $\pi_{n}(X, x)$, are defined by replacing loops at $x$ by continuous maps from $[0,1]^{n}$ to $X$ that associate the boundary of the $n$-cube to $x$. The product on such maps is then defined by gluing two faces of the $n$-cubes:

$p \cdot q\left(t_{1}, \ldots, t_{n}\right)= \begin{cases}p\left(2 t_{1}, t_{2}, \ldots, t_{n}\right) & \text { if } t_{1} \in\left[0, \frac{1}{2}\right], \\ q\left(2 t_{1}-1, t_{2}, \ldots, t_{n}\right) & \text { if } t_{1} \in\left[\frac{1}{2}, 1\right] .\end{cases}$

Conventionally, the set of path-connected components of $X$ is denoted by $\pi_{0}(X, x)$, but it has no group structure.

A continuous map $f: X \rightarrow Y$ is a weak homotopy equivalence if the morphisms $f_{n}: \pi_{n}(X, x) \rightarrow \pi_{n}(Y, y)$ defined by $f_{n}([p])=[f \circ p]$ are all bijective ( $f_{0}$ is just a bijection, not a morphism). Two spaces $X, Y$ are weakly homotopy equivalent if there is a sequence of spaces $X_{0}=X, X_{1}, \ldots, X_{r}=$ $Y(r \geqslant 1)$ such that there exist weak homotopy equivalences $X_{i-1} \rightarrow X_{i}$ or $X_{i} \rightarrow X_{i-1}$ for all $i \in[1, r]$. Two homotopy equivalent spaces are weakly homotopy equivalent. The converse is not true in general but Whitehead's theorem [28] implies that it is true for all spaces that are geometric realisations of simplicial or cubical complexes.

Two weakly homotopy equivalent spaces $X, Y$ have isomorphic homotopy groups. However, a weak homotopy equivalence is much more than a collection of isomorphisms between homotopy groups. On Figure 4 , we have depicted two cubical 3-complexes $X$ and $Y$ such that $Y \subset X$. Their geometric realisations have the same homotopy type and, therefore, are weakly homotopy equivalent. Nevertheless, it is clear that the inclusion $i: Y \rightarrow X$ is not a weak homotopy equivalence for it associates non-contractible loops to contractible loops. Likely, in image processing, we would reject such a thinning. So, the nature of the weak homotopy equivalence is an important information.

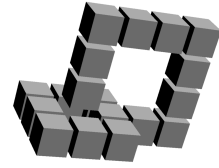

(a)

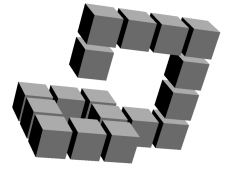

(b)
Fig. 4 (From [29]) (a) A cubical 3-complex $X$. (b) A subcomplex $Y$. Their geometric realisations have the same homotopy type. However, the inclusion $i: Y \rightarrow X$ is not a weak homotopy equivalence.

There is a case in which the weak homotopy equivalence reduces to the knowledge of the homotopy groups. When a set is weakly homotopy equivalent to a point, then it is connected and all its homotopy groups are trivial. Thus, obviously, any constant map is a weak homotopy equivalence. Such a space is said to be homotopically trivial. There are spaces that are homotopically trivial and that are not contractible as shown on Figure 5

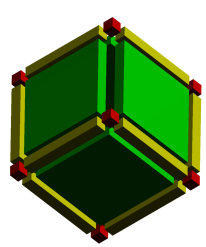

Fig. 5 A set of points (in red), closed lines (in yellow) and closed squares (in green) of $\mathbb{R}^{3}$ whose union forms a hollow cube with a fence. Equipped with the inclusion, this set is a finite topological space (see below Subsection 2.4 that is homotopically trivial but not contractible (the reader will be able to establish the proofs of these two assertions after the reading of Subsections 2.5 and 2.6.

\subsection{Complexes}

We do not recall definitions about simplicial complexes which are generally well known. The reader who whishes to recollect such a notion, or any one rapidly exposed below, is invited to find complementary information in a lecture book on algebraic topology, e.g. [30,31,32,33,34]. In digital images, grids are often cubic ones. It is then convenient, in image analysis, to replace simplices in complexes by $n$-cubes. 
As cubical complexes are not commonly used, we recall hereafter the main basic definitions (see also [23]). We set $\mathbb{F}_{0}^{1}=\{\{a\} \mid a \in \mathbb{Z}\}$ and $\mathbb{F}_{1}^{1}=\{\{a, a+1\} \mid a \in \mathbb{Z}\}$. A subset $f$ of $\mathbb{Z}^{n}$ which is the Cartesian product of $m$ elements of $\mathbb{F}_{1}^{1}$ and $n-m$ elements of $\mathbb{F}_{0}^{1}$ is a face or an $m$-face $\left(o f \mathbb{Z}^{n}\right), m$ is the dimension of $f$, and we write $\operatorname{dim}(f)=m$. We denote by $\mathbb{F}_{m}^{n}$ the set composed of all $m$-faces of $\mathbb{Z}^{n}$ and by $\mathbb{F}^{n}$ the set composed of all faces of $\mathbb{Z}^{n}$. Let $f \in \mathbb{F}^{n}$ be a face. The set $\left\{g \in \mathbb{F}^{n} \mid g \subseteq f\right\}$ is a cell and any union of cells is an abstract cubical complex. The geometric cubical complexes are defined in the same manner, except that we change the definition of $\mathbb{F}_{1}^{1}$ by setting $\mathbb{F}_{1}^{1}=\{[a, a+1] \mid a \in \mathbb{Z}\} \subset \mathbb{R}^{n}$. The geometric realisation $|K|$ of a geometric cubical complex $K$ is the union of its faces. Figure 6 illustrates these definitions.

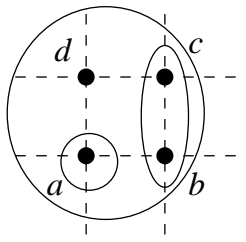

(a)

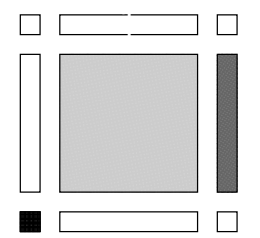

(b)
Fig. 6 (a) Four points in $\mathbb{Z}^{2}, a=(i, j), b=(i+1, j), c=(i+1, j+1)$, $d=(i, j+1)$. The faces $f=\{a\}, g=\{b, c\}=\{i+1\} \times\{j, j+1\}$ and $h=\{a, b, c, d\}=\{i, i+1\} \times\{j, j+1\}$ are symbolically depicted with ellipses. (b) Another (more semantic) symbolic representation, often used in this article. In black, the 0 -face $f$. In dark grey, the 1 -face $g$. In light grey, the 2-face $h$.

Whitehead [21] (an easier reference for modern readers is [34]) has defined elementary transformations on complexes as follows. Let $X$ be a complex (simplicial or cubical) and $(x, y)$ a pair of faces in $X$ such that $x$ is the only face of $X$ including $y$ (i.e., $X \backslash\{x, y\}$ is still a complex). Then, $(x, y)$ is a free pair, and the set $Y=X \backslash\{x, y\}$ is an elementary collapse of $X$, or $X$ is an elementary expansion of $Y$. If a set $Y$ is obtained from $X$ by a sequence of elementary collapses (a sequence of elementary collapses and expansions), then $Y$ is a collapse of $X$ ( $X$ and $Y$ are simple-homotopy equivalent or $X$ and $Y$ have the same simple-homotopy type) and one write $X \searrow Y(X \frown Y)$. A set is collapsible if it collapses onto a singleton.

If $Y$ is a collapse of $X$ then $|Y|$ is a strong deformation retract of $|X|$ and thus $|X|$ and $|Y|$ are homotopy equivalent [21]. Figure 7illustrates this property. In particular, if the complex is collapsible, its geometric realisation is contractible. The converse is not true as shown by the thin version of Bing's house with two rooms [26] or by Zeeman's dunce hat [27].
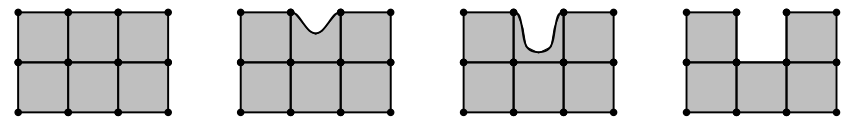

Fig. 7 (a) A complex $X$. (d) A complex $Y$ which is an elementary collapse of $X$. (b-c) Two steps in a strong deformation retraction of $|X|$ onto $|Y|$.

\subsection{Partially ordered sets}

The motivation for considering partially ordered sets (or posets) comes from (i) the observation that digital images are essentially finite (even when they are defined on $\mathbb{Z}^{n}$ to avoid difficulties on boundaries), (ii) that finite topological spaces of interest have the $T_{0}$-separation property 3 but not the $T_{1}$ separation property 4 (otherwise either some points could not be distinguished from a topological viewpoint or the space is totally disconnected), and (iii) that $\mathrm{T}_{0}$-spaces in which any intersection of open sets is an open set (as in finite spaces) are posets [35, 36] (this point is developed in Section 2.4).

Let $X$ be a set. A binary relation on $X$ is a partial order if it is reflexive, antisymmetric, and transitive. A partially ordered set, or poset, is a couple $(X, \leq)$ where the relation $\leq$ is a partial order on $X$. The relation $\geq$, defined on $X$ by $x \geq y$ iff $y \leq x$, is a partial order on $X$ called the dual order. We say that two points $x, y$ in $X$ are comparable if $x \leq y$ or $y \leq x$. If, for all pairs $(x, y)$ of elements of $X, x$ and $y$ are comparable, the relation $\leq$ is a total order on $X$. We write $x<y$ when $x \leq y$ and $x \neq y$ and we set:

$$
\begin{aligned}
& -x^{\uparrow}=\{y \in X \mid x \leq y\} \text { and } x^{\uparrow \star}=x^{\uparrow} \backslash\{x\}=\{y \in X \mid x<y\} ; \\
& \text { - } x^{\downarrow}=\{y \in X \mid y \leq x\} \text { and } x^{\downarrow \star}=x^{\downarrow} \backslash\{x\}=\{y \in X \mid y<x\} .
\end{aligned}
$$

If $x$ and $y$ are comparable, we write $x \asymp y$; otherwise, we write $x \neq y$. The set of points comparable with a given point $x$ is denoted $x^{\downarrow}\left(x^{\downarrow}=x^{\downarrow} \cup x^{\uparrow}\right)$, and we set $x^{\uparrow \star}=x^{\uparrow} \backslash\{x\}=$ $x^{\downarrow \star} \cup x^{\uparrow \star}$. A point $x \in X$ is minimal if $x^{\downarrow}=\{x\}$ and maximal if $x^{\uparrow}=\{x\}$. A point $x \in X$ is the minimum of $X$ if $x^{\uparrow}=X$ and is the maximum of $X$ if $x^{\downarrow}=X$. We say that a poset is locally finite if for each point $x$ in $X$, there are finitely many points comparable with $x$. A chain in $X$ is a totally ordered subset of $X$. The length of a chain is its cardinality minus one. The length of a poset $X$ is the maximal length of a chain in $X$ if such a maximum exists.5. The height of a point $x \in X$, denoted ht( $(x)$, is the length of $x^{\downarrow}$. If $x<y$ and there is no

3 A space has the $\mathrm{T}_{0}$-separation property if for any pair of distinct points there exists an open set that contains one of them and not the other.

${ }^{4}$ A space has the $T_{1}$-separation property if for any ordered pair of distinct points there exists an open set that contains the first of them and not the other. Now, let $x$ be a point in a finite $\mathrm{T}_{1}$-space $X$. For each $y \in X, y \neq x$, there exists an open neighbourhood of $x, U_{y}$, not containing $y$. Hence, $\{x\}=\bigcap U_{y}$ is open, that is to say, the topology on $X$ is the discrete topology in which all subsets are both open and closed and the only connected sets are the singletons.

5 Some authors define the length of a chain as its cardinality and the the maximal length of a chain in $X$ is also called the height of $X$. 


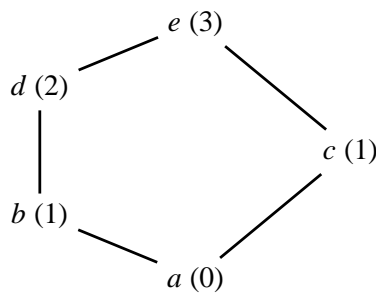

Fig. 8 The Hasse diagram of a poset defined by the set $\{a, b, c, d, e\}$ equipped with the order $\{(a, a),(a, b),(a, c),(a, d),(a, e)$, $(b, b),(b, d),(b, e),(c, c),(c, e),(d, d),(d, e),(e, e)\}$. Between parentheses, we give the height of the points. The length of this poset is 3 .

$z$ such that $x<z<y$, we say that $y$ covers $x$ and we write $x<y$. The Hasse diagram of the relation $\leq$ is the oriented graph of the relation $<$. When orienting all arcs from bottom to top, this diagram offers a good visual representation of (small) posets (see Figure 8).

Simplicial or cubical complexes equipped with the inclusion, $\subseteq$, or its dual, $\supseteq$, are locally finite posets. Moreover, for all $n \in \mathbb{N},\left(\mathbb{F}^{n}, \supseteq\right)$ is order isomorphic to $\left(\mathbb{F}^{n}, \subseteq\right.$ ) (that is, the combinatorial properties of the $k$-faces of $\mathbb{F}^{n}$ are equal to the ones of the $(n-k)$-faces if we replace $\subseteq$ by $\supseteq)$. Note that it is not true for simplicial complexes.

We extend to posets Whitehead's definitions of free pairs and collapses. A pair $(x, y)$ in a poset $X$ is a (combinatorial) free pair if $x$ is the only point (strictly) less than $y$ in $X$. If $(x, y)$ is a free pair in $X$, the set $X \backslash\{x, y\}$ is a collapse of $X$. When we can "thin" a subset $Y$ of $X$ to a subset $Z$ of $X$ by withdrawal of free pairs, we write $Y \searrow Z$.

\subsection{A-spaces}

A topological space $X$ is an A-space if any intersection of open sets is an open set. In such a space, closed sets satisfy the definition properties of open sets $(\emptyset, X$ are closed sets, any union and any intersection of closed sets is a closed set), so one can exchange open and closed sets. The obtained topology is then called the dual topology. As any set has a closure, any element $x$ of an A-space has a smallest neighbourhood (an open set included in any open set containing $x$ ), denoted by $U_{x}$, which is the closure of $\{x\}$ for the dual topology. Conversely, a topological space $X$ in which each point has a smallest neighbourhood is an A-space.

A $T_{0} A$-space is an A-space that has the $\mathrm{T}_{0}$-separation property (i.e., for any two distinct points $x, y$, there exists a neighbourhood containing just one of them). McCord has proved in [37] that if an A-space is not $\mathrm{T}_{0}$, the identification of the points that share the same smallest neighbourhood leads to a homotopy equivalent quotient space which is $T_{0}$.

There exists a canonical link between $\mathrm{T}_{0} \mathrm{~A}$-spaces and posets, established by Alexandroff.
Theorem 1 ([35]) Let $X$ be an $T_{0} A$-space. The relation $\leq$ defined on $X$ by $x \leq y$ if $x \in U_{y}$ is a partial order on $X$. Conversely, let $(X, \leq)$ be a poset. The set $U$ defined by $U=$ $\left\{U \subseteq X \mid \forall x \in U, x^{\downarrow} \subseteq U\right\}$ is a topology on $X$, the poset $X$ equipped with this topology is an $T_{0} A$-space and, for all $x \in X, U_{x}=x^{\downarrow}$.

Indeed, the choice to set $x \leq y$ if $x \in U_{y}$ is purely arbitrary. We could set $x \leq y$ if $y \in U_{x}$ and in literature both settings can be found (for instance, the choice $x \leq y$ if $y \in U_{x}$ is made by [35,38] and the other choice by [37,39,40,41]).

If $Y$ is a subset of $X$, the topology associated to the poset $(Y, \leq)$ is the topology induced by the one associated to the poset $(X, \leq)$. The dual topology of the topology associated to the poset $(X, \leq)$ is the topology associated to the dual order $\geq$.

From now on, posets will always be equipped with the topology $\mathcal{U}$ described in Theorem 1 . This topology leads to a nice characterisation of continuous maps.

Property 2 ([39]) Let $X, Y$ be posets. A function $f: X \rightarrow Y$ is continuous iff it is non-decreasing.

In the sequel, we will often have to test if a poset is contractible. Remember that a space is contractible if it has the homotopy type of a point, that is, if there exists a continuous map $H: X \times[0,1] \rightarrow X$ such that $H(x, 0)=x$ for any $x \in X$ and $x \mapsto H(x, 1)$ is a constant map. Intuitively, a set is contractible if it can be continuously shrunk to a point. Nevertheless, this intuition is of little help in a finite space. For instance, consider a geometric cubical complex $X$ composed of a closed unit square of $\mathbb{R}^{2}$, together with all its faces. Say, it is the one depicted on Figure 9 a). This complex is collapsible by $X \searrow X \backslash\{a, b\} \searrow\{d, e, f, h, i\} \searrow\{e, f, i\} \searrow\{i\}$. Since each elementary collapse is associated to a strong deformation retract in the Euclidean space $\mathbb{R}^{n}$, the realisation of this unit square is contractible and one can actually continuously schrink the square following the above sequence of collapses (which first step is the one illustrated on Figure 77. Now, this complex, equipped with the inclusion, is also a poset (the Hasse diagram of which is depicted on Figure 9(b)). Hence, $X$ is not only a combinatorial structure but also a topological space. However, we cannot follow the same steps to continuously shrink $X$ as before. For instance, we cannot remove continuously the face $\{a\}$ from $X \backslash\{b\}$ for there does not exist a non-decreasing function from $X \backslash\{b\}$ onto $X \backslash\{a, b\}$. Furthermore, in [25], we have shown that if $x, y$ are two faces in $\mathbb{F}^{n}(n \geq 3)$ such that $y \subset x$, the poset $(\{z \subset x \mid z \neq y\}, \subseteq)$, which looks like a sphere with a hole, is not contractible when $\operatorname{dim}(y) \leq \operatorname{dim}(x)-2$. This is clearly counterintuitive.

Hopefully, even if we have to build a new intuition to deal with finite spaces, there exist very easy properties like the following one which provides a sufficient condition to 


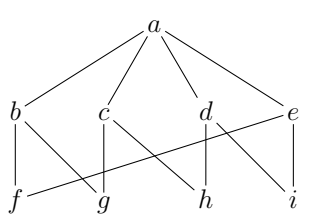

(b)

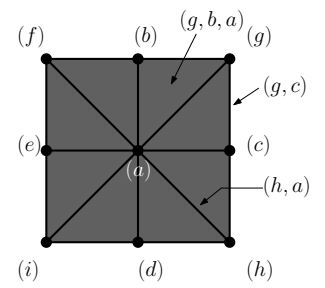

(c)

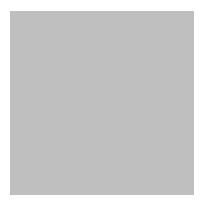

(d)

Fig. 9 (a) An abstract cubical cell $a^{\uparrow}$ which models a digital point of $\mathbb{Z}^{2}$. (b) The Hasse diagram of $X\left(a^{\uparrow}\right)$. (c) The simplicial complex $\mathcal{K}\left(X\left(a^{\uparrow}\right)\right)$. (d) The geometric realisation of $\mathcal{K}\left(X\left(a^{\uparrow}\right)\right)$.

guarantee the contractibility of a finite poset (a proof can be found, e.g., in [40, Lemma 6.2]; this is also a straightforward consequence of [39, Corollary 3]).

Property 3 Let $X$ be a poset. If $X$ has a maximum, or a minimum, then $X$ is contractible. In particular, for any $x \in$ $X, x^{\downarrow}$ and $x^{\uparrow}$ are contractible. Moreover, for any $x \in X, x^{\uparrow}$ is contractible.

There is a close link between posets and simplicial complexes, discovered by Alexandroff [35]. Let $X$ be a poset. The points in $X$ are the vertices of a simplicial complex $\mathcal{K}(X)$, the simplices of which are the finite chains of $X$ (see Figure 9). Conversely, it is plain that the simplices of a given simplicial complex $K$, equipped with the inclusion relation, form a locally finite poset, denoted $X(K)$.

These correspondences are not only algebraic, indeed the topologies are concerned as well. The following theorem, due to McCord, establishes the key properties of the map $\varphi_{X}:|\mathcal{K}(X)| \rightarrow X$ which associates to each point in the geometric realisation of $\mathcal{K}(X)$, the highest element of the unique open simplex it belongs to (remember that a simplex of $\mathcal{K}(X)$ is a chain).

Theorem 4 ([37, Theorem 2]) Let $X$ be a poset. There is a weak homotopy equivalence $\varphi_{X}:|\mathcal{K}(X)| \rightarrow X$. Furthermore, one can associate to each continuous map $f: X \rightarrow Y$ between two posets, the simplicial map $|\mathcal{K}(f)|$ such that the following diagram is commutative.

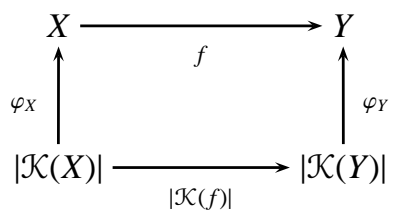

As the complex $\mathcal{K}(X)$ does not change if we consider the dual order on $X$, Theorem 4 implies that $(X, \leq)$ is weakly homotopy equivalent to $(X, \geq))$.

In the sequel of this section we direct our interest towards minimal deformations of the posets which do not alter their topology. To better understand the differences between the notions described below, we will take the same example all along the three next subsections. Consider the space $\mathbb{F}^{3}$ as defined in Subsection 2.2 The set $\mathbb{F}^{3}$ together with inclusion is obviously a poset. Let $x_{0}$ be a 3 -face in $\mathbb{F}^{3}$ and $x_{1}$ be a face in $x_{0}^{\downarrow \star}$. We set $X_{0}=\mathbb{F}^{3} \backslash\left\{x_{0}\right\}$ and $X_{1}=X_{0} \backslash\left\{x_{1}\right\}$. Our goal is to shrink $X_{0}$ onto $X_{1}$.

\subsection{Unipolar points}

The significance of unipolar points in posets was discovered by Stong [39] in the 60's and later rediscovered by Bertrand [38]. Most results in this subsection were first established in Stong's article for finite spaces but can be easily adapted to any posets.

Definition 5 (Unipolar point) Let $X$ be a poset. A point $x \in$ $X$ is:

- down unipolar if $x^{\downarrow \star}$ has a maximum;

- up unipolar if $x^{\uparrow \star}$ has a minimum;

- unipolar if it is either down unipolar or up unipolar.

Property 6 ([39, Proof of Theorem 2] and [25, Proposition 4]) Let $(X, \leq)$ be a poset. A point $x \in X$ is unipolar iff $X \backslash\{x\}$ is a strong deformation retract of $X$.

Definition 7 (Core) Let $(X, \leq)$ be a poset. Let $Y \subseteq X$ be a subset of $X$. We say that $Y$ is a core of $X$ if the poset $(Y, \leq)$ has no unipolar point and it is a strong deformation retract of $X$.

\section{Property 8 ([39, Theorems 2, 4])}

1. Any finite poset has a core and two cores of the same poset are homeomorphic.

2. Two finite posets are homotopy equivalent iff they have homeomorphic cores.

Observe in particular that Property 8 implies that one can greedily remove the unipolar points of a finite poset in order to obtain a core which will be homeomorphic to any other core of the same poset. In particular, when the poset is contractible, we have the following corollary.

Corollary 9 ([25, Corollary 4]) If $X$ is finite and contractible, there is a sequence $\left(x_{i}\right)_{i=0}^{r}(r \geq 0)$ of points in $X$ such that $X=\left\{x_{i}\right\}_{i=0}^{r}$ and, for all $j \in[1, r], x_{j}$ is unipolar in $\left\{x_{i}\right\}_{i=0}^{j}$. Furthermore, if $x \in X$ is unipolar, we can choose $x_{r}=x$. 
As an unipolar point in a poset $(X, \leq)$ is, clearly, also an unipolar point in the poset $(X, \geq)$, one can easily deduce from Corollary 9 and Property 6 the following corollary.

Corollary 10 Let $(X, \leq)$ be a finite poset. Then, $(X, \leq)$ is contractible iff $(X, \geq)$ is contractible.

Thanks to the next Property, one can build well balanced shrinking algorithms by deleting unipolar points with same heights in parallel.

Property 11 ([38, Property 3] and [25, Proposition 5]) If $x \neq y$ are unipolar points, then either (a) $y$ is unipolar in $X \backslash\{x\}$, or (b) for one order on $X(\leq$ or $\geq)$, $x$ is down-unipolar and covers $y$, for the other order $y$ is down-unipolar and covers $x$ and the map $\varphi: X \backslash\{x\} \rightarrow X \backslash\{y\}$ defined by $\varphi(z)=z$ if $z \neq y$ and $\varphi(y)=x$ is an homeomorphism.

Example 12 Let us consider the test set $X_{0}$, described at the end of Subsection 2.4 It is plain that the 2-faces of $x_{0}$ are up unipolar in $X_{0}$. Thus, if $\operatorname{dim}\left(x_{1}\right)=2$, the set $X_{1}$ is a strong deformation retract of $X_{0}$. If $\operatorname{dim}\left(x_{1}\right) \leq 1, x_{1}$ is not unipolar so $X_{1}$ is not a strong deformation retrac 6 of $X_{0}$.

This example shows us that unipolar points are not enough "powerful" to be used in thinning or growing procedures. This is the reason why we introduce now $\beta$-simple points.

\section{$2.6 \beta$-simple points}

The notion of $\beta$-simple points was first introduced by Bertrand 7 in [38] in order to define topologically sound thinning algorithms in posets. In his article, Bertrand uses a specific definition for the homotopy type. On the other hand, Barmak and Minian [41] gives the same definition in the classical framework in order to perform a collapse operation in posets which actually corresponds to the collapse operation in complexes associated to posets.

Definition $13(\beta$-simple point) Let $(X, \leq)$ be a poset. A point $x \in X$ is:

- down $\beta$-simple (in $X$ ) if $x^{\downarrow \star}$ is contractible;

- up $\beta$-simple (in $X$ ) if $x^{\uparrow \star}$ is contractible;

- $\beta$-simple (in $X$ ) if it is either down $\beta$-simple or up $\beta$ simple.

From this definition and Corollary 10 , we straightforwardly infer the next proposition.

${ }^{6}$ In fact, it is easy to prove that $X_{1}$ is not even a retract of $X_{0}$ since $x_{1}$ belongs to, at least, 9 connected pairs in $X_{0}$ and any function from $X_{0}$ to $X_{1}$, equal to identity on $X_{1}$, will disconnect one of these pairs.

${ }^{7}$ Bertrand calls the up $\beta$-simple points, $\alpha$-simple points, and the down $\beta$-simple points, $\beta$-simple points where $\alpha$ and $\beta$ denote the order and its dual in the poset $X$.
Proposition 14 Let $(X, \leq)$ be a poset. Let $x$ be a $\beta$-simple point in $X$. Then $x$ is $\beta$-simple in $X$ equipped with the reverse order and the dual topology.

Unipolar points are $\beta$-simple points since if $x \in X$ is a down (resp. up) unipolar point, $x^{\downarrow \star}$ (resp. $x^{\uparrow \star}$ ) has a maximum (resp. minimum) and is therefore contractible (Property 3). We saw previously (Property 6) that the removal of a unipolar point is a strong deformation retraction. It is no longer true for $\beta$-simple points (see our test set $X_{0}$ of Example 12 with $\operatorname{dim}\left(x_{1}\right) \leq 1$ for a counterexample). Nevertheless, the next property states that homotopy groups are not changed by such a deletion and, furthermore, the following theorem ensures that this deletion corresponds to a strong deformation retraction on the continuous analogue.

Property 15 ([41, Proposition 3.3]) Let $X$ be a finite poset. Let $x \in X$ be a $\beta$-simple point. Then, the inclusion map $i$ : $X \backslash\{x\} \rightarrow X$ is a weak homotopy equivalence.

Theorem 16 ([41, Theorem 3.10]) Let $X$ be a finite poset. Let $x \in X$ be a $\beta$-simple point and $\mathcal{K}(X), \mathcal{K}(X \backslash\{x\})$ the simplicial complexes associated to $X$ and $X \backslash\{x\}$, respectively. Then, $\mathcal{K}(X)$ collapses onto $\mathcal{K}(X \backslash\{x\})$.

From an algorithmic point of view, like unipolar points, $\beta$-simple points have good properties since they can be deleted in parallel. Obviously, if $x, y$ are two points in $X$ with $\operatorname{ht}(x)=\operatorname{ht}(y)$, there is no need to know whether $x$ has been deleted from $X$ or not to decide if $y^{\downarrow \star}$, or $y^{\uparrow \star}$ is contractible. Moreover, as we have seen above, the decision on the contractibility can be greedily performed. Thus, a topology-preserving thinning procedure in a poset $X$ of finite length $\ell$ consists of repeating until stability the removal of the $\beta$ simple points of height $k$ for $k=0$ to $\ell$.

Example 17 Let us consider once again the test set $X_{0}$. If $\operatorname{dim}\left(x_{1}\right)=2$, we have already seen that $x_{1}$ is unipolar, so it is also $\beta$-simple. If $\operatorname{dim}\left(x_{1}\right)=1$, the Hasse diagram of $x_{1}^{\uparrow \star}$ in the poset $X_{0}$ is an acyclic graph composed of the four 2faces of $\mathbb{F}^{3}$ including $x_{1}$ and the three 3-faces of $\mathbb{F}^{3}$ including $y$ and distinct from $x_{0}$. Thus, it is contractible and $x_{1}$ is up $\beta$-simple. The inclusion map $i_{1}: X_{1} \rightarrow X_{0}$ is therefore a weak homotopy equivalence. If $\operatorname{dim}\left(x_{1}\right)=0$, let $y_{0}, y_{1}, y_{2}$ be the three 2-faces including $x_{1}$ and included in $x_{0}$. The reader can check in Figure 10 that these three faces are up-unipolar in $x_{1}^{\uparrow \star}$ and that $x_{1}^{\uparrow \star} \backslash\left\{y_{0}, y_{1}, y_{2}\right\}$ is a core of $x_{1}^{\uparrow \star}$. Hence, $x_{1}^{\uparrow \star}$ is not contractible and $x_{1}$ is not $\beta$-simple.

\section{$2.7 \gamma$-simple points}

The example set $X_{0}$ highlights the need for a weaker condition on points to be deleted when processing a thinning in a 


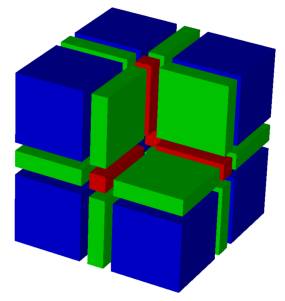

(a)

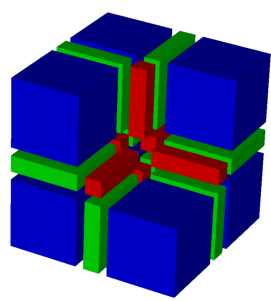

(b)
Fig. 10 (a) The subset $x_{1}^{\uparrow \star}$ of $X_{0}$. (b) The core of $x_{1}^{\uparrow \star}$ in $X_{0}$.

digital image. The following definition of $\gamma$-simple point 8 and their properties are due to Barmak and Minian [42]. Bertrand [38] defines a quite similar notion.

Property 18 leads to an alternative definition of $\beta$-simple points: a point $x$ is $\beta$-simple iff $x^{\uparrow \star}$ is contractible. In turn, this alternative definition leads to the definition of $\gamma$-simple points.

Property 18 ([42, Proposition 3.3]) Let $X$ be a finite poset and $x$ a point in $X$. Then $x^{\uparrow \star}$ is contractible iff $x^{\downarrow \star}$ or $x^{\uparrow \star}$ is contractible.

Definition 19 A point $x$ of a poset is a $\gamma$-simple point if the poset $x^{\text {} \star}$ is homotopically trivial.

As we have observed (see Subsection 2.4) that the homotopy groups of a poset are unchanged if we consider the reverse order on $X$, we can state the following proposition.

Proposition 20 Let $X$ be a finite poset and $x$ be a $\gamma$-simple point in $X$. Then $x$ is $\gamma$-simple in $X$ equipped with the reverse order and the dual topology.

Since a contractible space is obviously homotopically trivial, a $\beta$-simple point is a $\gamma$-simple point. In general, the converse is false as it will appear in Example 24. Nevertheless, if the length of $X$ is less than or equal to 2 (intuitively, if $X$ is 2-dimensional), then any $\gamma$-simple point is a $\beta$-simple point [42].

The following property gives a sufficient condition for a point to be $\gamma$-simple. This condition enables to decrease the cost of looking for $\gamma$-simple points since the length of $x^{\uparrow \star}$ or $x^{\downarrow \star}$ is always less than or equal to the length of $x^{\downarrow \star}$.

Property 21 ([42, Proposition 3.17]) Let $X$ be a finite poset and $x$ a point in $X$. Then $x^{\downarrow \star}$ is homotopically trivial if $x^{\downarrow \star}$ or $x^{\uparrow \star}$ is homotopically trivial.

If the length of the space is less than or equal to 3 , and $x$ is neither a maximum nor a minimum, the height of $x^{\uparrow \star}$ and

${ }^{8}$ Barmak and Minian call them $\gamma$-points. To be consistent with the previous subsection, we prefer to call them $\gamma$-simple points. $x^{\downarrow \star}$ is less than or equal to 1 . Hence, if $x^{\uparrow \star}$ or $x^{\downarrow \star}$ is homotopically trivial, it is contractible. Thanks to Property 18 , we deduce that $x^{\downarrow \star}$ is contractible and therefore homotopically trivial.

The next property ensures that the deletion of a $\gamma$-simple point does not modify the homotopy groups.

Property 22 ([42]) ([42, Proposition 3.10]) Let $X$ be a finite poset. Let $x \in X$ be a $\gamma$-simple point. Then, the inclusion $i: X \backslash\{x\} \rightarrow X$ is a weak homotopy equivalence.

Finally, the following theorem states that, when deleting a $\gamma$-point in a finite poset, the homotopy type of the continuous analogue is unchanged.

Theorem 23 ([42]) ([42], Theorem 3.15]) Let $X$ be a finite poset and let $x \in X$ be a $\gamma$-simple point. Then $|\mathcal{K}(X \backslash\{x\})|$ and $|\mathcal{K}(X)|$ are simple-homotopy equivalent.

In a 3D image $X$, the cost to decide whether the set $x^{\uparrow \star}$ is

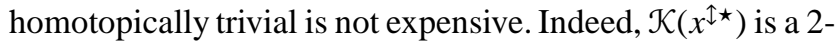
dimensional simplicial complex and it is enough to compute its connected components and its Euler characteristic. An alternative to look at $\gamma$-simple points, in any dimension, is to remove $\beta$-simple points in $x^{\uparrow \star}$ until stability. If the result is a singleton, by Property $15, x^{\uparrow \star}$ is weak homotopy equivalent to a point and therefore homotopically trivial. Moreover, the scheme proposed for the deletion of simple points is still valid ( $\gamma$-simple points with same height can be removed in parallel).

Example 24 Let us consider the test set $X_{0}$. We have seen that $x_{1}$ is a $\beta$-simple point iff $\operatorname{dim}\left(x_{1}\right) \geq 1$. Suppose now that $\operatorname{dim}\left(x_{1}\right)=0$. The chain complex $\mathcal{K}\left(x_{1}^{\uparrow \star}\right)$ (see Subsection 2.4) is depicted in Figure 11] in a $2 D$-space and in a $3 D$-space. It is clearly contractible, so $x^{\uparrow \star}$ is homotopically trivial (Theorem (4). Thus, $x_{1}$ is a $\gamma$-point and the injection $i: X_{1} \rightarrow X_{0}$ is a weak homotopy equivalence.

\subsection{Complexes and simplicity}

In this subsection, we establish some specific properties of spaces of cubical or simplicial complexes. The proofs of these new results are provided in Appendices $B$ and $C$

In Section 4, the proof of Theorem 47 needs the space to have a property that can be understood in the framework of complexes as asking the boundary of a cell with a "large hole" to be homotopically trivial. So, we introduce the following definition.

Definition 25 A poset $X$ has the pierced sphere property if, for any $x, y \in X$ such that $y$ covers $x$, the set $x^{\uparrow \star} \backslash\{y\}$ is homotopically trivial. 


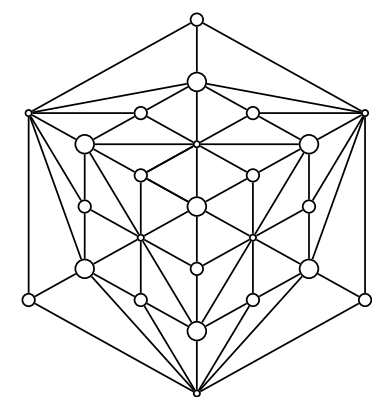

(a)

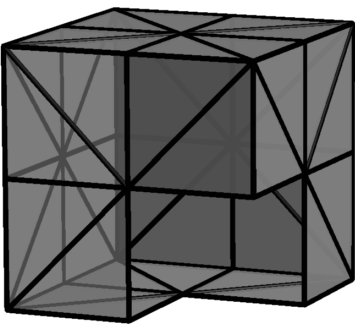

(b)
Fig. 11 (a) The pure simplicial 2-complex $\mathcal{K}\left(x_{1}^{\uparrow \star}\right)$ in a 2D space. The large/middle/small circles are vertices associated to 3-/2-/1-faces of $x_{1}^{\uparrow \star}$. (b) The complex $\mathcal{K}\left(x_{1}^{\uparrow \star}\right)$ in a $3 \mathrm{D}$ space. The seven vertices associated to the 3 -faces of $x_{1}^{\uparrow \star}$ are in corner position and the vertices associated to the 1 -faces are in centre position.

The next proposition states that this pierced sphere property is satisfied by the spaces of cubical or simplicial complexes. In Appendix B, we actually prove an extended version of this statement (Proposition 58).

Proposition 26 Let $X$ be a cubical or a simplicial complex equipped with the order $\supseteq$. Then, $X$ has the pierced sphere property.

In digital topology, the usual requirement for a point $y$ to be simple for an object $Y$ in a space $X$ (that is a point which can be removed from $Y$ in a topologically sound thinning procedure) is that (i) the inclusion $i: Y \backslash\{y\} \rightarrow Y$ induces a one-to-one correspondence between the connected components of the object before and after the removal (i.e., $Y$ and $Y \backslash\{y\})$, (ii) the inclusion $i^{\prime}: X \backslash Y \rightarrow(X \backslash Y) \cup\{y\}$ induces a one-to-one correspondence between the connected component of the background before and after the removal (i.e., $X \backslash Y$ and $X \backslash Y \cup\{y\}$ ), (iii) the inclusion $i$ induces isomorphisms between the fundamental groups of the connected components of the object before and after the removal, (iv) the inclusion $i^{\prime}$ induces isomorphisms between the fundamental groups of the connected components of the background before and after the removal [43]. In [29], it has been proved, thanks to the linking number borrowed to knots theory, that for 3D digital images interpreted with the $(6,26)$ or the $(26,6)$ pair of adjacencies, there is no need to consider the fundamental groups of the background since their preservation is implied by the three first conditions. The following theorem generalises, in our framework, this property to spaces of any dimension (and, in a certain sense, defined in [22], for any pair of adjacencies).

Theorem 27 Let $X$ be a cubical complex equipped with the order $\supseteq$ which is also a cubical complex for the dual order $\subseteq$. Let $Y$ be a proper subset of $X$ and $y$ be a $\beta$-simple point in $Y$. Then $y$ is $\gamma$-simple in $(X \backslash Y) \cup\{y\}$.

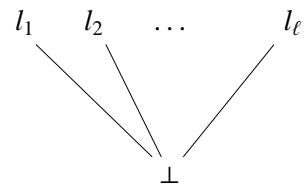

Fig. 12 Hasse diagram of the label set $L=\left\{l_{i}\right\}_{i=1}^{\ell} \cup\{\perp\}$.

Remark 28 We do not know if this theorem remains true in any dimension if we replace the hypothesis " $y$ is a $\beta$-simple point" by " $y$ is a $\gamma$-simple point". Nevertheless, if the dimension of $X$ is $2, \gamma$-simple points are $\beta$-simple points, so it is obviously true in this case. Moreover, we have proved, by checking all configurations with the help of a computer program, that it is also true in $\mathbb{F}^{3}$, the space of 3-dimensional cubical complexes. In Appendix D Counterexample 61 provides a case where Theorem 27 is false when the space $X$ is not a complex for the dual order.

\section{Label images}

Let $L$ be a finite poset with a minimal element, denoted $\perp$, and such that two distinct elements in $L \backslash\{\perp\}$ are not comparable. We set $L^{\star}=L \backslash\{\perp\}$ and we write $\ell$ for the cardinality of $L^{\star}$. The elements of $L^{\star}$ are called proto-labels. The Hasse diagram of the poset $L$ is depicted in Figure 12 A label digital image is a function defined on $\mathbb{Z}^{n}$, with values in $L$, and equal to $\perp$ everywhere except on a finite set of points of $\mathbb{Z}^{n}$.

Let $l \in L, l \neq \perp$ be a proto-label and $\lambda$ a label digital image. The set $\lambda^{-1}(\{l\})$ is the support of the proto-label $l$ (in the label digital image $\lambda$ ). The union of the supports of all proto-labels is the domain of the image $\lambda$. (This domain is finite by definition.) The set $\lambda^{-1}(\{\perp\})$ is the background of the image $\lambda$. The background and the supports define a partition of $\mathbb{Z}^{n}$.

In order to equip the discrete grid on $\mathbb{Z}^{n}$ with a topology, we enrich this grid by adding low dimensional points between the xels of $\mathbb{Z}^{n}$ (for instance, in $\mathbb{Z}^{3}$, we add surfels, linels and pointels) whose purpose is to link the distinct adjacent xels and to confer a poset structure to the discrete space. Typically, such a space is the space of cubical complexes, $\mathbb{F}^{n}$, or any poset associated to a cellular decomposition of the space [44, 45, 46, 47, 48]. Thereby, the label digital images considered in this article are defined on a locally finite poset $(X, \leq)$ : we wish to link points of $\mathbb{Z}^{n}$ to finitely many neighbours. Indeed, all sets $x^{\uparrow \star}$ and $x^{\downarrow \star}$ which appear in the definitions of $\beta / \gamma$-simple points will be finite. This will allow us to use the results of Section 2 .

Furthermore, we suppose that the embedding of $\mathbb{Z}^{n}$ in $X$ puts in one-to-one correspondence the points of $\mathbb{Z}^{n}$ with the minimal points of $X$. The reader must be aware that this is counterintuitive. For instance, if the poset is the space of cubical complexes, $\mathbb{F}^{n}$, this one must be ordered by the 


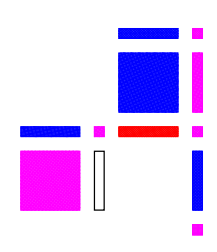

(a)

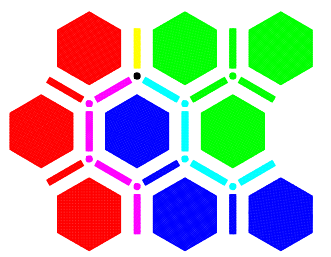

(b)

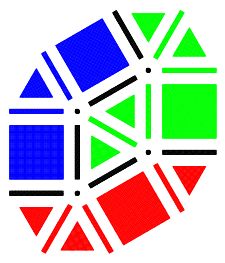

(c)
Fig. 13 Label images. The proto-labels are $r, g, b$ (depicted in red, green and blue). The other labels are obtained by using the additive colour model (e.g., $\{r, b\}$ is depicted in magenta) except $T$ which is depicted in black ( $\perp$ is depicted in white). (a) $X$ is a subset of $\mathbb{F}^{2} . T$ is the power set $2^{\{b, r\}}$. Observe that in this image, there are points of height 0 that have distinct dimensions. (b) $X$ is built from an hexagonal tessellation. $T$ is the power set $2^{\{r, g, b\}}$. The labels of the points of height greater than 0 are assigned according to the rule which will be used in Section 5 a label is the supremum of the labels of the minimal points in the neighbourhood. (c) $X$ is built from a semi-regular tessellation. The labels are given according to the same rule as in (b) but $T$ is not a power set: $T=\{\perp, r, g, b, \top\}$.

dual of the inclusion, $\supseteq$, i.e., the height of a face is its codimension. The reason to do so is to put the xels of $\mathbb{Z}^{n}$, which contain all the information of the original image, at the same height in the poset, namely "on the floor". Then, we can add, above those minimal points, the topological "glue" that is needed to interpret the image. Most of the time, the labels of the minimal points will be proto-labels, or $\perp$, that is minimal labels in $T$ and the image will be non-decreasing. In the sequel, we identify the points of $\mathbb{Z}^{n}$ with their images in $X$ so the $x e l s$ are the minimal points of $X$.

Since we enrich the initial space with low dimensional faces in order to get both a topological space and an algebraic structure, we are led to do so with the label set to extend the digital label image on these supplementary low dimensional faces. That is why we embed the label set in an atomistic lattice $(T, \leq)$ whose minimum is the embedding of $\perp$ and atoms are the embeddings of the proto-labels of $L$ (a few definitions and properties about lattices can be found in Appendix $\mathrm{A}$. In the sequel, we identify the elements of $L$ with their images in $T$. We denote by $T$ the maximum of $T$. A label is an element of $T$. Given a (proto-)label set $L^{\star}$ the smallest lattice $T$ including $L$ is $T=L \cup\{T\}$. This is the lattice used by Ronse and Agnus in [49,50] to define morphological operators on label images. The largest atomistic lattice in which we can embed $L$ is the power set $2^{L^{\star}}$ (with the natural embedding which associates $\emptyset$ to $\perp$ and the singleton $\{l\}$ to any proto-label $l$ ).

Some ways to associate labels to points in $X$ that are not xels are discussed in [44,51]. We have proposed, in [22], our own modus operandi to embed a binary digital image defined on $\mathbb{Z}^{n}$ in a binary image defined on $\mathbb{F}^{n}$. It can be straightforwardly extended to label images and we use it, in a particular case, in Section 5 but we do not develop more

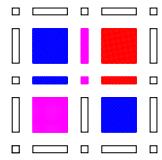

(a)

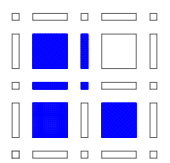

(b)

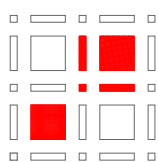

(c)

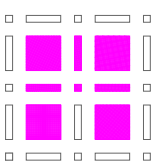

(d)
Fig. 14 (a) A label image whose domain is $\mathbb{F}^{2}$ and whose codomain is the power set $T=2^{\{b, r\}}=\{\emptyset,\{b\},\{r\},\{b, r\}\}$ equipped with the inclusion. The points with label $\{b\}$ are depicted in blue, those with label $\{r\}$ in red and those with label $\{b, r\}$ in magenta. The points of the background (label $\perp=\emptyset$ ) are depicted in white with a black border or are not depicted. (b) In blue, the support of the label $\{b\}$. (c) In red, the support of the label $\{r\}$. (d) In magenta, the support of $T=\{b, r\}$.

this issue in this article. This is why we actually just set the following definition for label images.

Definition 29 (Label images) Let $X$ be a locally finite poset and $T$ an atomistic lattice. A label image is a function $\mu$ : $X \rightarrow T$.

Figure 13 provides various examples of label images.

We have seen that when we start from a label digital image $\lambda: \mathbb{Z}^{n} \rightarrow L$ and we construct a label image $\mu: X \rightarrow T$, the labels of the minimal points of $X$ (i.e., the xels) are the atoms of $T$ (i.e., the proto-labels). When a label image has this property, we say that this image is pure.

A label image can be seen as a superposition of binary layers. Indeed, if $\mu$ is a label image, and $l \in L^{\star}$ is a protolabel, the image $\mu_{l}=\mu \wedge l$ is a binary image whose codomain is $\{\perp, l\}$ (remember that we denote by $\wedge$ and $\vee$ the infimum and supremum operations of the lattice $T$ : see Appendix $\mathrm{A}$. The next proposition establishes that $\mu$ is the supremum of all the binary images $\mu_{l}, l \in L^{\star}$.

Proposition 30 Let $\mu: X \rightarrow T$ be a label image. Let $L^{\star}$ be the set of atoms of $T$. Then, $\mu=\bigvee_{l \in L^{\star}} \mu_{l}$ where, for all $l \in L^{\star}, \mu_{l}=\mu \wedge l$.

Proof We set $L^{\star}=\left\{l_{i}\right\}_{i=1}^{\ell}$. Let $x$ be a point in $X$. Let $A \subseteq L^{\star}$ be the set of atoms in $T$ which are less than or equal to $\mu(x)$. Then, $\mu(x)=\bigvee_{a \in A} a$ for $T$ is atomistic. Let $l \in L^{\star}$, be an atom in $T$. We have $(\mu \wedge l)(x)=\mu(x) \wedge l=\left(\bigvee_{a \in A} a\right) \wedge l$. It is plain that $(\mu \wedge l)(x)=l$ iff $l \in A$ and $(\mu \wedge l)(x)=\perp$ iff $l \notin A$. Thus, $\mu(x)=\bigvee_{a \in A} a=\bigvee_{l \in A}(\mu(x) \wedge l)=\bigvee_{l \in L^{\star}}(\mu(x) \wedge l)=$ $\bigvee_{l \in L^{\star}}(\mu \wedge l)(x)$.

Let $\mu: X \rightarrow T$ be a label image and $t$ be a label. The support of $t$ in $\mu$ is the subset $\langle t\rangle_{\mu}$ of $X$ equal to $\{x \in X \mid$ $\mu(x) \wedge t \neq \perp$. When there is no ambiguity, we also say the support of $t$ instead of the support of $t$ in $\mu$ and we write 


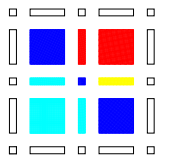

(a)

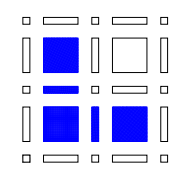

(b)

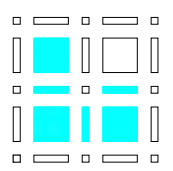

(c)

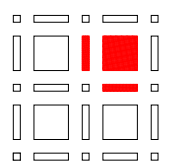

(d)

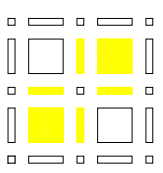

(e)
Fig. 15 (a) A label image $\mu$ whose domain is $\mathbb{F}^{2}$ and whose codomain is the power set $T=2^{\{r, g, b\}}$ equipped with the inclusion. The labels are depicted as in Figure 14 plus the labels $\{g, b\},\{r, g\}$ which are depicted respectively in cyan and yellow. The point $x$ is the 0 -face at the centre of the figure. We have $\mu(x)=\{b\}$. We want to test if the point $x$ is simple for the label $t=\{r\}$ (that is, we want to know if we can label the point $x$ with $t$ while keeping unchanged the topology of the supports of the $2^{3}-1$ non-minimal labels in $T$ ). There are two labels $u$ such that $u \wedge \mu(x) \neq \perp$ and $u \wedge t=\perp:\{b\}$ and $\{g, b\}$. (b) In blue, the set $x^{\downarrow \star} \cap\langle\{b\}\rangle$ which is contractible. (c) In cyan, the set $x^{\downarrow \star} \cap\langle\{g, b\}\rangle$ which is contractible. Hence, the first half of the test (namely, condition $(i)$, in Definition 31] succeeds. Now, let us consider the labels $u$ for which $u \wedge \mu(x)=\perp$ and $u \wedge t \neq \perp:\{r\}$ and $\{r, g\}$. (d) In red, the set $x^{\downarrow \star} \cap\langle\{r\}\rangle$ which is contractible. (e) In yellow, the set $x^{\downarrow \star} \cap\langle\{r, g\}\rangle$ which is not contractible (it has two connected components). The second half of the test (namely, condition (ii), in Definition 31 then fails. Therefore, the point $x$ is not simple for the label $\{r\}$. Giving the label $\{r\}$ to $x$ would connect two distinct components of the label $\{r, g\}$.

$\langle t\rangle$ instead of $\langle t\rangle_{\mu}$. The support of a proto-label (in a label image) is the subset of $X$ whose points have a label greater or equal to this proto-label. The support of a label $t \neq \perp$ is the union of the supports of the proto-labels in $t^{\downarrow}: x \in$ $\langle t\rangle \Leftrightarrow \mu(x) \wedge t \neq \perp \Leftrightarrow \exists t_{i}$ atom,$t_{i} \leq \mu(x) \wedge t \Leftrightarrow \exists t_{i} \leq$ $t$ atom, $x \in\left\langle t_{i}\right\rangle$. The support of the label $\perp$ is the empty set. The cosupport of $t$ in $\mu$ (or the cosupport of $t$ ) is the complement in $X$ of the support of $t$ in $\mu$. We denote it by $\langle t\rangle_{\mu}^{c}$ or $\langle t\rangle^{c}$. Figure 14 illustrates these definitions.

We have seen in Section 1 that in a label digital image in which one wants to preserve the topological properties inside the supports of the proto-labels and between these supports, it is important, when performing a change of label on a point, to maintain the topology of any union of supports of proto-labels. In other words, we have to preserve the topology of any set identified by a proto-label in the partition of the space associated to the initial digital label image but also of any set defined by any coarser partition of the space. In the proposed framework, the supports (of the labels) are subsets of $X$ that are exactly the unions of the supports of the proto-labels. Hence, the supports of the labels in $T$ are the sets for which we must ensure the topological invariance. This has lead us to the following definition, exemplified in Figure 15

Definition 31 (Simple point for a label) Let $\mu: X \rightarrow T$ be a label image. Let $t \in T$ be a label. A point $x \in X$ is a simple point for (the label) $t$ if the following two conditions are verified:

(i) for all labels $u \in T$ such that $u \wedge \mu(x) \neq \perp$ and $u \wedge t=\perp$, $x$ is $\beta$-simple for the set $\langle u\rangle$;

(ii) for all labels $u \in T$ such that $u \wedge \mu(x)=\perp$ and $u \wedge t \neq \perp$, $x$ is $\beta$-simple for the set $\langle u\rangle \cup\{x\}$.

In this definition, the first condition, $u \wedge \mu(x) \neq \perp$ and $u \wedge t=$ $\perp$, means that $x$ is in the support of $u$ in $\mu$ but it will no more be in it if the image is modified by giving the label $t$ to $x$. Conversely, the second condition, $u \wedge \mu(x)=\perp$ and $u \wedge t \neq \perp$, means that $x$ is not in the support of $u$ in $\mu$ but it will be in it if the image is modified by giving the label $t$ to $x$. In each case, by requiring $x$ to be $\beta$-simple for the sets $\langle u\rangle_{\mu}$, we ensure that there exists a weak homotopy equivalence between each support before and after the modification of the image $\mu$ and, if $X=\mathbb{F}^{n}$, the cosupports will also be weakly homotopic (see Proposition 34). Remember that this implies also that the operation corresponds to strong deformation retractions on the realisations of the simplicial complexes associated to these supports (Theorem 16). In a binary image (i.e., with $T=\{\perp, T\}$, Definition 31 comes down to require $x$ to be $\beta$-simple in $\langle T\rangle$ or $x$ to be $\beta$-simple in $\langle T\rangle \cup\{x\}$, depending on $x$ is in the object or in the background. Observe also that any point in $\mu^{-1}(t)$ is simple for the label $t$.

Since the poset $X$ is locally finite, the sets $x^{\uparrow \star}$ and $x^{\downarrow \star}$ are finite. Therefore, according to Corollary 9, one can test the simplicity of a point $x \in X$ by greedily removing unipolar points in the sets $x^{\uparrow \star} \cap Y$ and $x^{\downarrow \star} \cap Y$ where $Y=\langle u\rangle$ or $Y=\langle u\rangle \cup\{x\}$, for all $u \in T$. When the lattice $T$ is distributive, the following proposition allows us to speed up this test by reducing temporarily the size of $T$ by identifying the atoms of $T$ not "involved" in $\mu\left(x^{\downarrow}\right)$ with the label $\perp$. Observe that if the lattice $T$ is distributive and $\mu$ is defined from a label digital image $\lambda: \mathbb{Z}^{n} \rightarrow L$ as suggested in the introduction of Section 3 , then $T$ is a finite, atomistic and distributive lattice whose atoms are identified with the elements of $L^{\star}$, that is $T$ is the power set $2^{L^{\star}}$ (see Appendix $\mathrm{A}$ ).

Proposition 32 Let $\mu: X \rightarrow T$ be a label image. Let $t$ be a label and $x$ be a point in $X$. Let $L^{\star}$ be the set of atoms in $T$ and $L_{x}$ be the subset of $L^{\star}$ whose elements are less than or equal to an element of $\mu\left(x^{\uparrow}\right)$. Let $\varphi: T \rightarrow T$ be the function that maps any label $u$ onto the label $\varphi(u)=\bigvee\left\{a \in L_{x} \mid a \leq\right.$ $u\}$.

(i) If the point $x$ is simple for $t$ in $\mu$ then $t \in \varphi(T)$ and $x$ is simple for $t$ in the image $\varphi \circ \mu: X \rightarrow \varphi(T)$.

(ii) Conversely, if the lattice $T$ is distributive, $t \in \varphi(T)$, and $x$ is simple for $t$ in the image $\varphi \circ \mu$, then the point $x$ is simple for $t$ in $\mu$.

Proof ( $i$ ) We assume that $x$ is simple for $t$ in $\mu$. Let us suppose that $t \notin \varphi(T)$. Then it must exist an atom $a \notin L_{x}$ such 
that $a \leq t$ (otherwise $t=\varphi(t) \in \varphi(T)$ ). This label $a$ is such that $a \wedge \mu(x)=\perp$ (by definition of $a$ and $L_{x}$ ) and $a \wedge t \neq \perp$. But $x$ cannot be $\beta$-simple for the set $\langle a\rangle_{\mu} \cup\{x\}$ since $x^{\uparrow \star} \cap\langle a\rangle_{\mu}$ is empty (by definition of $a$ and $L_{x}$ ). Thus, we have a contradiction with the simplicity of $x$ for $t$ in $\mu$. So $t \in \varphi(T)$. Let $u$ be a label in $\varphi(T)$ such that $u \wedge \varphi(\mu(x)) \neq \perp$ and $u \wedge t=\perp$. Since, trivially, $\varphi$ reduces to identity on $\mu\left(x^{\mathfrak{}}\right)$, we have that $u \wedge \mu(x)=u \wedge \varphi(\mu(x)) \neq \perp$. As $x$ is simple for $t, u \wedge \mu(x) \neq \perp$ and $u \wedge t=\perp$, it comes that $x$ is $\beta$-simple for the set $\langle u\rangle_{\mu}$. We have already observed that the images $\mu$ and $\varphi \circ \mu$ are equal on $x^{\downarrow}$. Note, moreover, that the $\beta$-simplicity only involves a subset of $x^{\uparrow}$. Hence, $x$ is $\beta$-simple for the set $\langle u\rangle_{\varphi \circ \mu}$. Similarly, when $u$ is a label in $\varphi(T)$ such that $u \wedge \varphi(\mu(x))=\perp$ and $u \wedge t \neq \perp$, we deduce as above that $x$ is $\beta$-simple for the set $\langle u\rangle_{\varphi \circ \mu} \cup\{x\}$. We can then conclude that $x$ is simple for $t$ in $\varphi \circ \mu$.

(ii) We now assume that $x$ is simple for $t$ in $\varphi \circ \mu$ with $t \in \varphi(T)$. Let $u$ be a label in $T$ such that $u \wedge \mu(x) \neq \perp$ and $u \wedge t=\perp$. By the very definition of $L_{x}$, we have $u \wedge$ $\mu(x)=\varphi(u \wedge \mu(x))$. Thus $\varphi(u \wedge \mu(x)) \leq u$ and $\varphi(u \wedge \mu(x)) \leq$ $\mu(x)$. Then, since $\varphi$ is an opening (see Appendix A), we have $\varphi(u \wedge \mu(x)) \leq \varphi(u)$ and $\varphi(u \wedge \mu(x)) \leq \varphi(\mu(x))$. Thereafter, $u \wedge \mu(x)=\varphi(u \wedge \mu(x)) \leq \varphi(u) \wedge \varphi(\mu(x))$. Thus, $\varphi(u) \wedge \varphi(\mu(x)) \neq$ $\perp$. We have also $\varphi(u) \wedge t=\varphi(u) \wedge \varphi(t)$, since an opening is idempotent and $t \in \varphi(T)$, and $\varphi(u) \wedge \varphi(t) \leq u \wedge t$, since an opening is anti-extensive. Thus, we get $\varphi(u) \wedge t=\perp$. As $x$ is simple for $t$ in $\varphi \circ \mu, \varphi(u) \wedge \varphi(\mu(x)) \neq \perp$ and $\varphi(u) \wedge t=\perp$, we derive that $x$ is $\beta$-simple for the set $\langle\varphi(u)\rangle_{\varphi \circ \mu}$ which implies that $x$ is $\beta$-simple for the set $\langle u\rangle_{\mu}$ (for $x^{{ }^{\uparrow \star}} \cap\langle u\rangle_{\mu}=x^{\uparrow \star} \cap$ $\left.\langle\varphi(u)\rangle_{\varphi \circ \mu}\right)$. When $u$ is a label in $T$ such that $u \wedge \mu(x)=\perp$ and $u \wedge t \neq \perp$, we derive that $\varphi(u) \wedge \varphi(\mu(x))=\perp$ (with similar arguments as above). Let us now assume that $T$ is distributive. It can easily be seen that $t \in \varphi(T)$ implies that $u \wedge t \in \varphi(T)$ (any atom less than or equal to $t$ is in $L_{x}$ ). Thus, $\perp \neq u \wedge t=\varphi(u \wedge t) \leq \varphi(u) \wedge \varphi(t)=\varphi(u) \wedge t$. We conclude, as previously, that $x$ is $\beta$-simple for $\langle u\rangle_{\mu} \cup\{x\}$. Hence, $x$ is simple for $t$ in $\mu$.

In Appendix D Counterexample 62 illustrates the fact that Proposition 32 is generally false when the lattice is not distributive.

Let us now define the topological properties we want to preserve when processing a label image.

Definition 33 Let $\mu, v: X \rightarrow T$ be two label images.

- If, for all labels $t \in T,\langle t\rangle_{\mu}$ and $\langle t\rangle_{\nu}$ are weak homotopy equivalent, we say that these images are equivalent and we write $\mu \approx \nu$.

- If, furthermore, $\langle t\rangle_{\mu}^{c}$ and $\langle t\rangle_{\nu}^{c}$ are weak homotopy equivalent for all labels $t$, we say that the images are strongly equivalent.

We write $\mu+(x, t)$ for the image equal to $\mu$ except in $x$, where its value is $t$.
Based on these definitions, we have the following result.

Proposition 34 Let $\mu: X \rightarrow T$ be a label image. Let $x$ be a simple point for the label $t$. Then, $\mu$ and $\mu+(x, t)$ are equivalent, strongly equivalent if $X=\mathbb{F}^{n}$.

Proof Let $v$ be the image $\mu+(x, t)$. Let $u$ be a label. By definition of the image $v$, the supports $\langle u\rangle_{\mu}$ and $\langle u\rangle_{\nu}$ are equal, except possibly on $x$. Therefore, if $(u \wedge \mu(x)=\perp$ and $u \wedge t=\perp)$ or $(u \wedge \mu(x) \neq \perp$ and $u \wedge t \neq \perp)$, then $\langle u\rangle_{v}=\langle u\rangle_{\mu}$. In the other cases, from Definition 31, $x$ is $\beta$-simple for $\langle u\rangle_{\mu}$ (if $x \in\langle u\rangle_{\mu}$ ) or $x$ is $\beta$-simple for $\langle u\rangle_{\mu} \cup\{x\}$ (if $x \notin\langle u\rangle_{\mu}$ ). Hence, $\langle u\rangle_{\nu}$ is weak homotopy equivalent to $\langle u\rangle_{\mu}$. If $X=\mathbb{F}^{n}$, we derive from Theorem 27 that $x$ is $\gamma$-simple for $\langle u\rangle_{\mu}^{c}$ (if $x \in\langle u\rangle_{\mu}$ ) or $x$ is $\gamma$-simple for $\langle u\rangle_{\mu}^{c} \cup\{x\}$ (if $x \notin\langle u\rangle_{\mu}$ ). Thus, $\langle u\rangle_{\nu}^{c}$ and $\langle u\rangle_{\mu}^{c}$ are weak homotopy equivalent (Property 22).

The next proposition is an easy consequence of Definitions 13 and 31. From a practical point of view, it is quite important since it allows us to define parallel thinning (or growing) algorithms in label image by simultaneously modifying the label of simple points with the same height.

Proposition 35 Let $\mu_{0}: X \rightarrow T$ be a label image. Let $t \in T$ be a label. Let $Y=\left\{y_{i}\right\}_{i=0}^{k}(k \geq 1)$ be a set of points with the same height, simple for the label $t$. For all $i \in[1, k]$, we set $\mu_{i}=\mu_{i-1}+\left(y_{i-1}, t\right)$. Then, for all $i \in[0, k], y_{i}$ is a simple point for the label t in $\mu_{i}$.

Figure 16 provides an example of label thinning/growing by giving the label $t$ to simple points for $t$, processing points with same height during the same pass on the image.

\section{Closed support images}

In this section, we focus on digital images that could be associated to digital images considered with the $\left(3^{n}-1,2 n\right)$ adjacency pair in $n \mathrm{D}$ cubic grids (namely $\mathbb{Z}^{n}$ ). This adjacency pair corresponds to the adjacency of closed objects of the continuous space [52] and has therefore led us to investigate label images in which the support of any label is a closed set. Hence, we define a closed support (label) image as a label image whose supports are closed sets (for any label). The following proposition establishes that the closed support label images are the non-decreasing maps from $X$ onto $T$ (that is the continuous maps from $X$ to $T$ (Property 2)).

Proposition 36 Let $\mu: X \rightarrow T$ be a label image. The supports of the labels in $\mu$ are closed sets iff $\mu$ is a non-decreasing function from $(X, \leq)$ to $(T, \leq)$. 


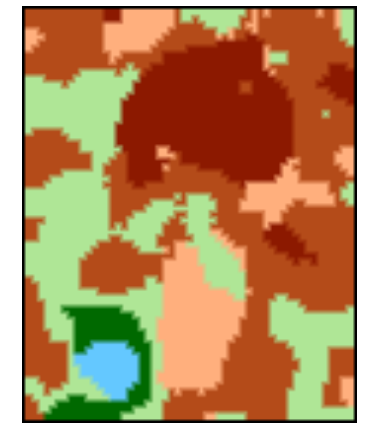

(a)

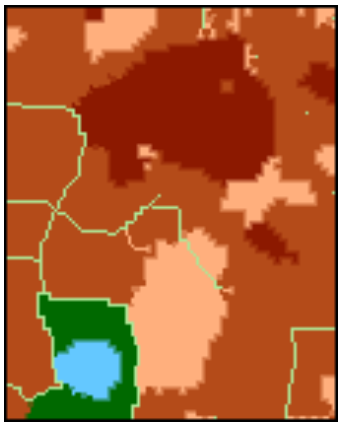

(b)

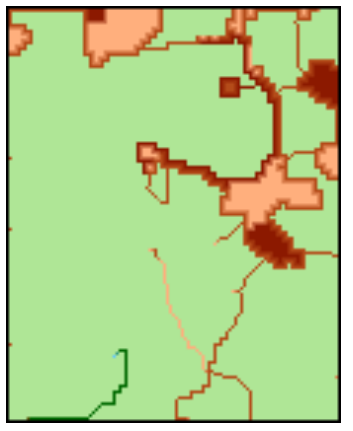

(c)
Fig. 16 (a) A label image defined from $\mathbb{F}^{2}$ to $T=2^{L^{\star}}$ where $L^{\star}$ contains 6 proto-labels. (b) The light green label has been shrunk by removing simple points in its support, dimension by dimension, until stability. (c) The same label has been expanded by adding to its support simple points, dimension by dimension, until stability. In each case ((b) and (c)), we claim that the process maintains the topology (namely, the weak homotopy type) of the $63\left(2^{6}-1\right)$ supports and 63 cosupports that are defined by the initial partition.

Proof Let us suppose that, for all $t \in T,\langle t\rangle$ is a closed set of $X$. Let $x, y$ be two points of $X$ such that $x \leq y$. If $\mu(x)=\perp$, obviously $\mu(x) \leq \mu(y)$. We assume now that $\mu(x) \neq \perp$. When we defined the supports, we have established that $\langle\mu(x)\rangle=$ $\bigcup_{t_{i} \in A}\left\langle t_{i}\right\rangle$ where $A$ is the set of atoms in $T$ that are less than or equal to $\mu(x)$. By definition of a support, $x \in\left\langle t_{i}\right\rangle$ for each atom $t_{i} \in A$. Thus, as the supports are closed and $x \leq y, y \in$ $\left\langle t_{i}\right\rangle$ for each atom $t_{i}$ such that $t_{i} \leq \mu(x)$. It means that each atom less than or equal to $\mu(x)$ is less than or equal to $\mu(y)$. Hence, as $T$ is atomistic, we have $\mu(x) \leq \mu(y)$.

Conversely, suppose that $\mu$ is non-decreasing. Let $t$ be a label and $x$ be a point in $\langle t\rangle^{c}$. Then, for all $y \in x^{\downarrow}$, we get $\mu(y) \leq \mu(x)$ (for $\mu$ is non-decreasing) and furthermore, $\mu(y) \wedge t \leq \mu(x) \wedge t=\perp$. Thereby, $y \in\langle t\rangle^{c}$. We conclude that $\langle t\rangle^{c}$ is an open set and therefore $\langle t\rangle$ is a closed set.

\subsection{Cut}

In Section 3 we have given sufficient conditions for an elementary modification of a label image to preserve the topology. However, if we want to work with closed support label

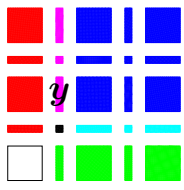

(a)

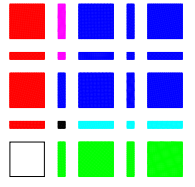

(b)
Fig. 17 (a) A closed support image $\mu: \mathbb{F}^{2} \rightarrow 2^{\{r, g, b\}}$. The labels are depicted as in Figure 13 The point $y$, which has a label $\mu(y)=\{r, b\}$, is simple for the label $\{r\}$. (b) The image $\mu+(y,\{r\})$ is no longer a closed support image.

images, we have to go further since, without improvement, these conditions fail to maintain closed supports as we can see in Figure 17

In the poset $X$, a set $F$ is closed iff for any point $x \in F$ the points greater than $x$ are also in $F$. This is like in a simplicial complex, where any subset of a face of the complex is also a face of the complex. It is well known that the set of simplicial complexes is closed under the collapse operation, which furthermore "preserves topology" [21]. So, we have adapted this notion to label images in order to maintain both the closedness and the topology of any label support. Roughly speaking, we have found that this goal can be achieved if we require the supports of some labels in the sub-poset $x^{\downarrow}$ to be contractible (where $x$ is the point whose label has to be modified).

Proposition 37 Let $\mu: X \rightarrow T$ be a closed support image. Let $x<y$ be two points in $X$. The following statements are equivalent.

(i) For all $u \in T$ such that $y \in\langle u\rangle, x^{\uparrow} \cap\langle u\rangle$ is contractible.

(ii) For all $u \in T$ such that $y \in\langle u\rangle$ and $x \notin\langle u\rangle$, $x$ is $\beta$-simple for $\langle u\rangle \cup\{x\}$.

(iii) The point $x$ is simple for the label $\mu(y)$.

Proof $(i) \Rightarrow$ (ii) Let $u$ be a label such that $y \in\langle u\rangle$ and $x \notin\langle u\rangle$. Then, $x^{\uparrow \star} \cap(\langle u\rangle \cup\{x\})=x^{\uparrow} \cap\langle u\rangle$ is contractible by hypothesis. So, $x$ is $\beta$-simple for the set $\langle u\rangle \cup\{x\}$ (Property 18 and Definition 13 .

(ii) $\Rightarrow$ (iii) First, we observe that, since $\mu$ is a closed support image, $\mu$ is non-decreasing (Proposition 36). Thus $\mu(x) \leq$ $\mu(y)$ and, therefore, $u \wedge \mu(x) \leq u \wedge \mu(y)$ for all $u \in T$. Thereafter, there does not exist any label $u$ such that $u \wedge \mu(x) \neq \perp$ and $u \wedge \mu(y)=\perp$. If $u$ is a label such that $u \wedge \mu(x)=\perp$ and $u \wedge \mu(y) \neq \perp$, by hypothesis, $x$ is $\beta$-simple for $\langle u\rangle \cup\{x\}$. Hence, $x$ is simple for $\mu(y)$.

(iii) $\Rightarrow$ (i) Let $u$ be a label such that $y \in\langle u\rangle$. Then either $x \in\langle u\rangle$ and, since the set $x^{\uparrow}$ is contractible in any poset (Property 3), in particular in the poset $\langle u\rangle, x^{\uparrow} \cap\langle u\rangle$ is contractible, or $x \notin\langle u\rangle$ and, by the hypothesis, $x$ is $\beta$-simple for $\langle u\rangle \cup\{x\}$, that is, $x^{\uparrow} \cap\langle u\rangle=x^{\uparrow \star} \cap(\langle u\rangle \cup\{x\})$ is contractible. 


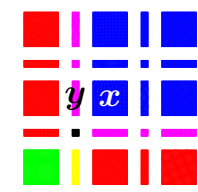

(a)

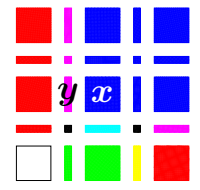

(b)

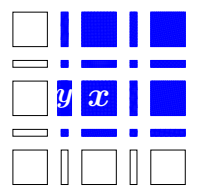

(c)
Fig. 18 Label images: $\mathbb{F}^{2} \rightarrow 2^{\{r, g, b\}}$. The labels are depicted as in Figure 13 (a) $(x, y)$ is a free pair for the label $\{b\}$ : the only label $u$ such that $y \in\langle u\rangle$ and $x \notin\langle u\rangle$ is $\{r\}$ and the set $x^{\uparrow \star} \cap\langle\{r\}\rangle$ is clearly contractible. (b) $(x, y)$ is not a free pair for $\{b\}$ since $\langle\{r\}\rangle \cap x^{\uparrow}$ is not connected (this set contains $y$, the 0 -face in magenta and the two 0 faces in black). (c) $(x, y)$ is free for the label $\{b\}$ (since here, there is no label $u$ such that $y \in\langle u\rangle$ and $x \notin\langle u\rangle$, Definition 38 reduces to the classical definition of a free pair in complexes).

Definition 38 (Free pair) Let $\mu: X \rightarrow T$ be a closed support image and $t \in T$ be a label. A pair $(x, y)$ of points in $\langle t\rangle$ is a free pair for the label $t$ if $x$ is the only point in $\langle t\rangle$ such that $x<y$ and the statements of Proposition 37 are satisfied by the pair $(x, y)$.

The label $t$ involved in Definition 38 cannot be the label $\perp$ since $\langle t\rangle$ contains at least the two points of the free pair and $\langle\perp\rangle=\emptyset$. We exemplify in Figure 18 the definition of free pairs.

The following proposition is the analogue of Proposition 32 for free pairs.

Proposition 39 Let $\mu: X \rightarrow T$ be a label image. Let $t$ be a label and $x, y$ be two points in $\langle t\rangle$. Let $L^{\star}$ be the set of atoms in $T$ and $L_{x}$ be the subset of $L^{\star}$ whose elements are less than or equal to an element of $\mu\left(x^{\uparrow}\right)$. Let $\varphi: T \rightarrow T$ be the function that maps the label $u$ onto the label $\varphi(u)=\bigvee\{a \in$ $\left.L_{x} \mid a \leq u\right\}$. If the pair $(x, y)$ is free for $t$ in $\mu$ then $(x, y)$ is free for $t$ in the closed support image $\varphi \circ \mu: X \rightarrow \varphi(T)$. Conversely, if the lattice $T$ is distributive and $(x, y)$ is a free pair for $t$ in the image $\varphi \circ \mu$, then the pair $(x, y)$ is free for $t$ in $\mu$.

Proof Before beginning the proof, note that the definition of $L_{x}$ is the same as in Proposition 32 though we have set $L_{x}=\left\{a \in L^{\star} \mid \exists y \in x^{\uparrow}, a \leq \mu(y)\right\}$ instead of $L_{x}=\left\{a \in L^{\star} \mid\right.$ $\left.\exists y \in x^{\uparrow}, a \leq \mu(y)\right\}$. Indeed, any atom $a$ less than or equal to an element $\mu(z), z \leq x$, is less than or equal to $\mu(x)$ since here $\mu$ is non-decreasing (Proposition 36). Thus, the two definitions coincide.

Now, suppose that $(x, y)$ is free for $t$ in $\mu$. Since $\varphi$ is an opening (see Appendix $\mathrm{A}$ ) and an opening is order-preserving, $\varphi \circ \mu$ is non-decreasing and is thus a closed support image (Proposition 36). Moreover, $\varphi$ is anti-extensive so $\mu(z) \wedge t=$ $\perp \Rightarrow \varphi \circ \mu(z) \wedge t=\perp$ and $\varphi$ reduces to identity on $\mu\left(x^{\downarrow}\right)$ so $\varphi(\mu(x))=\mu(x)$. Thus, $y^{\downarrow \star} \cap\langle t\rangle_{\varphi \circ \mu}=y^{\downarrow \star} \cap\langle t\rangle_{\mu}=\{x\}$. Now, from Proposition 32, we derive that $x$ is simple for the label $\mu(y)$ in the image $\varphi \circ \mu$. We conclude that $(x, y)$ is a free pair for $t$ in the image $\varphi \circ \mu$.
Conversely, suppose that the lattice $T$ is distributive and that $(x, y)$ is free for $t$ in the image $\varphi \circ \mu$. Since $\varphi(\mu(y))=\mu(y)$, we derive from Proposition 32, that $x$ is simple for the label $\mu(y)$ in the image $\mu$. Furthermore, let $z$ be a point in $y^{\downarrow \star}$ such that $\mu(z) \wedge t \neq \perp$. As $\mu$ is non-decreasing, any atom of $T$ less than or equal to $\mu(z)$ is less than or equal to $\mu(y)$. Thus, $\varphi(\mu(z))=\mu(z)$ and $\varphi(\mu(z)) \wedge t \neq \perp$. From the hypothesis we derive that $z=x$. Thereby, $y^{\downarrow \star} \cap\langle t\rangle_{\mu}=\{x\}$ and $(x, y)$ is a free pair for the label $t$ in $\mu$.

The definition of free pairs in a label image is an extension of the notion of free pair in complexes: if $X$ is a simplicial or cubical complex, $\mu: X \rightarrow T$ a label image and $(x, y)$ a free pair for the label $t$ in $\mu$, then $(x, y)$ is a free pair for the complex $\langle t\rangle_{\mu}$. The following proposition shows that Definition 38 reduces to the classical definition of a free pair when the two points in the pair share the same label.

Proposition 40 Let $\mu$ be a closed support image, $t \in T a$ label and $(x, y)$ a pair of points in $\langle t\rangle$. If $\mu(x)=\mu(y)$ and $y^{\downarrow \star} \cap\langle t\rangle=\{x\}$, then $(x, y)$ is a free pair for the label $t$.

Proof Since $\mu(x)=\mu(y)$, there is no label $u \in T$ such that $y \in\langle u\rangle$ and $x \notin\langle u\rangle$ so the statement (ii) of Proposition 37 is satisfied.

Proposition 41 Let $\mu$ be a closed support image, $t \in T a$ label and $(x, y)$ a free pair for $t$. Then, $x$ is a minimal element in $\langle t\rangle, y$ is down unipolar in $\langle t\rangle$ and $x<y$ in $X$.

Proof The point $x$ is a minimal element of $\langle t\rangle$ for $x$ is the only point in $\langle t\rangle$ such that $x<y$. The point $y$ is down unipolar for the same reason. Finally, as $\langle t\rangle$ is a closed set, $x^{\uparrow}$ is included in $\langle t\rangle$ and there does not exist any point in $\langle t\rangle$ between $x$ and $y$ for $y^{\downarrow} \cap\langle t\rangle=\{x, y\}$. Thereafter $x<y$.

The next definition introduces the notion of cut. Broadly speaking, a cut of the label $t$ in a closed support image $\mu$ consists of removing $t$ from a free pair $(x, y)$ for $t$. Indeed, in order to maintain the boundaries between supports, the label of $y$ must move towards the other points of $x^{\uparrow \star}$ and the labels "behind the boundary", i.e., the labels of $y^{\downarrow \star} \backslash\{x\}$, must replace $t$ on $\{x, y\}$. Figure 19 exemplifies this definition.

Definition 42 (Cut) Let $\mu: X \rightarrow T$ be a closed support image, $t \in T$ a label and $(x, y)$ a free pair for the label $t$. The label image $\mu_{y, t}: X \rightarrow T$ defined by:

$\mu_{y, t}(z)= \begin{cases}\bigvee_{z \in y \downarrow \star \backslash\{x\}} \mu(z) & \text { if } z \in\{x, y\} \\ \mu(z) \vee \mu(y) & \text { if } z \in x^{\uparrow \star} \backslash\{y\} \\ \mu(z) & \text { otherwise }\end{cases}$

is $a$ cut of $t$ at $y$ in $\mu$ (if $y^{\downarrow \star} \backslash\{x\}=\emptyset$, we $\left.\operatorname{set} \mu(x)=\mu(y)=\perp\right)$. 


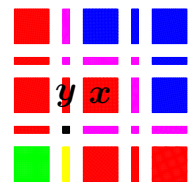

(b)

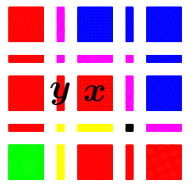

(c)

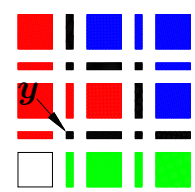

(a)

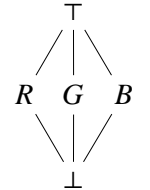

(b)

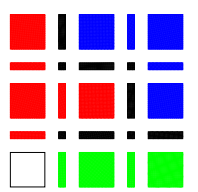

(c)
Fig. 19 (a) An image $\mu$ with a free pair $(x, y)$ for the label $\{b\}$ (see Figure 18 a)) (b) The cut $\mu^{\prime}=\mu_{y,\{b\}}$. Let $y^{\prime}$ be the 0 -face in black just below $y$ and $x^{\prime}$ the 1 -face in magenta just beneath $x$. The pair $\left(x^{\prime}, y^{\prime}\right)$ is free for $\{b\}$ in $\mu^{\prime}$. (c) The cut $\mu_{y^{\prime},\{b\}}^{\prime}$.

Figure 20 shows that cuts are of no interest in non-distributive lattices since it may happen that the label to be removed from a free pair is still present in the cut.

The notion of cut is an extension to label images of the notion of collapse for complexes. When $X$ is a simplicial or cubical complex and $T$ is distributive, the following proposition states that a cut for the label $t$ is a collapse for the support of $t$ and in particular, if $T=\{\perp, \top\}$, that is when $\mu$ is a binary image, a cut is nothing but a collapse.

Proposition 43 Let $\mu_{0}, \mu_{1}$ be two closed support images from the complex $X$ to the distributive lattice $T$ and $t \in T$ be a label. If $\mu_{1}$ is a cut of $\mu_{0}$ for $t$, then $\langle t\rangle_{\mu_{1}}$ is a collapse of $\langle t\rangle_{\mu_{0}}$.

Proof Let $\mu_{0}$ be a closed support image, $(x, y)$ a free pair of $\mu_{0}$ for the label $t$ and $\mu_{1}$ the cut $\mu_{y, t}$. From Definition 38 , the pair $(x, y)$ is free for the set $\langle t\rangle_{\mu_{0}}$ and from Definition 42. the supports of $t$ in $\mu_{0}$ and $\mu_{1}$ are equal except possibly in $x^{\uparrow}$. As $\mu_{0}$ is a closed support image, $x^{\uparrow}$ is included in $\langle t\rangle_{\mu_{0}}$. As $\mu_{1}(z)=\mu_{0}(z) \vee \mu_{0}(y)$ for all $z \in x^{\uparrow} \backslash\{x, y\}$, the set $x^{\uparrow} \backslash\{x, y\}$ is still included in $\langle t\rangle_{\mu_{1}}$. The label of the points $x, y$ in the image $\mu_{1}$ is $\bigvee_{z \in y^{\downarrow \star} \backslash\{x\}} \mu_{0}(z)$. Since $x$ is the only point in $\langle t\rangle_{\mu_{0}} \cap y^{\downarrow \star}$ and we assume $T$ to be distributive, we have $\mu_{1}(x) \wedge t=\mu_{1}(y) \wedge t=\bigvee_{z \in y \downarrow \star \mid\{x\}}\left(\mu_{0}(z) \wedge t\right)=\bigvee_{z \in y \downarrow \star \mid\{x\}} \perp=\perp$ Thus, neither $x$ nor $y$ is in $\langle t\rangle_{\mu_{1}}$ and $\langle t\rangle_{\mu_{1}}=\langle t\rangle_{\mu_{0}} \backslash\{x, y\}$. We conclude that the complex $\langle t\rangle_{\mu_{1}}$ is a collapse of $\langle t\rangle_{\mu_{0}}$.

When the lattice $T$ is distributive, the following proposition enables to specify which supports are modified by a cut. If the lattice $T$ is not distributive, this proposition fails (see Counterexample63 in Appendix D).

Proposition 44 Let $T$ be a distributive lattice and $\mu: X \rightarrow$ $T$ be a closed support image. Let $(x, y)$ be a free pair for the label $t \in T$ and $\mu_{y, t}$ the cut of t at $y$ in $\mu$. For any label $u \in T$ whose support does not contain $y$, we have $\langle u\rangle_{\mu_{y, t}}=\langle u\rangle_{\mu}$.

Proof Let $u$ be a label such that $y \notin\langle u\rangle_{\mu}$ and, since $\mu$ is a closed support image, $x \notin\langle u\rangle_{\mu}$. From Definition 42 $\mu_{y, t}(z)=$ $\mu(z)$ for any point $z \in\langle u\rangle_{\mu}$ not in $x^{\uparrow}$. Since $\mu$ is non-decreasing, $\mu(z) \leq \mu(y)$ for all $z<y$. Hence, $\bigvee_{z \in y^{\downarrow} \star \mid\{x\}} \mu(z) \leq \mu(y)$. Thus, $\mu_{y, t}(y) \wedge u=\mu_{y, t}(x) \wedge u=\left(\bigvee_{z \in y \bowtie \star}|\{x\}| \mu(z)\right) \wedge u \leq \mu(y) \wedge$ $u=\perp$, that is $x, y \notin\langle u\rangle_{\mu_{y, t}}$. Finally, for any point $z \in x^{\uparrow \star} \backslash\{y\}$,
Fig. 20 (a) A closed supports label image $\mu: X \rightarrow T$. The Hasse diagram of $T$ is depicted in (b) ( $T$ is not distributive). The labels $\perp, R, G, B, \top$ are depicted respectively in white, red, green, blue and black. (c) The cut $\mu^{\prime}=\mu_{y, B}$ which is equal to $\mu$.

$\mu_{y, t}(z)=\mu(z) \vee \mu(y)$ thus $z \in\langle u\rangle_{\mu_{y, t}}\left(\right.$ i.e., $\left.\mu_{y, t}(y) \wedge u \neq \perp\right)$ iff $z \in\langle u\rangle_{\mu}$ (for $T$ is distributive).

As stated at the beginning of this subsection, the main advantage of free pairs and cuts on simple points for labels is to enable to remain inside the set of closed support images when we modify a label image with topological constraints.

Proposition 45 Let $\mu: X \rightarrow T$ be a closed support image, $t \in T$ a label and $(x, y)$ a free pair for the label $t$. Then the cut $\mu_{y, t}$ is a closed support image.

Proof Let $(x, y)$ be a free pair for a label $t$ in a closed support image $\mu$. By hypothesis, $\mu$ is non-decreasing (Proposition 36). Let us prove that $\mu_{y, t}$ is also non-decreasing. Let $a, b$ be two points in $X$ such that $a<b$ and, thereafter, such that $\mu(a) \leq \mu(b)$. The proof is made by exhaustion.

- If $b \notin x^{\uparrow}$ then $a \notin x^{\uparrow}$. Then $\mu_{y, t}(b)=\mu(b)$ and $\mu_{y, t}(a)=$ $\mu(a)$. In this case, obviously, we have $\mu_{y, t}(a) \leq \mu_{y, t}(b)$.

- If $b \in\{x, y\}$ and $a \notin x^{\uparrow}$ then $a \in y^{\downarrow \star} \backslash\{x\}$ and $\mu_{y, t}(a)=$ $\mu(a) \leq \bigvee_{z \in y^{\star \star} \backslash\{x\}} \mu(z)=\mu_{y, t}(b)$. Thus, $\mu_{y, t}(a) \leq \mu_{y, t}(b)$.

- If $a, b \in\{x, y\}$ then $\mu_{y, t}(a)=\mu_{y, t}(b)$.

Note that it is impossible to have $b \in\{x, y\}$ and $a \in$ $x^{\uparrow} \backslash\{x, y\}$ for $x<y$ in $X$ (Proposition 41).

- If $b \in x^{\uparrow} \backslash\{x, y\}$ and $a \notin x^{\uparrow}$ then $\mu_{y, t}(a)=\mu(a) \leq \mu(b) \leq$ $\mu(b) \vee \mu(y)=\mu_{y, t}(b)$.

- If $b \in x^{\uparrow} \backslash\{x, y\}$ and $a \in\{x, y\}, \mu_{y, t}(a)=\bigvee_{z \in y \downarrow \downarrow \mid\{x\}} \mu(z) \leq$ $\mu(y)$ (for $\mu$ is non-decreasing) and $\mu(y) \leq \mu(b) \vee \mu(y)=$ $\mu_{y, t}(b)$. Thereby, $\mu_{y, t}(a) \leq \mu_{y, t}(b)$.

- If $a, b \in x^{\uparrow} \backslash\{x, y\}$, then $\mu_{y, t}(a)=\mu(a) \vee \mu(y)$ and $\mu_{y, t}(b)=$ $\mu(b) \vee \mu(y)$ thus $\mu_{y, t}(a) \leq \mu_{y, t}(b)$.

In each possible case, we have $\mu_{y, t}(a) \leq \mu_{y, t}(b)$. Hence, $\mu_{y, t}$ is non-decreasing.

When a label image $\mu$ is obtained from a label digital image (defined on $\mathbb{Z}^{n}$ ) by the procedure we have described at the beginning of Section 4 this image is pure $(\mu(x)$ is an atom, or $\perp$, for any xel $x$ ). Cuts preserve purity under an hypothesis which is satisfied, for example, by pseudomanifolds (see, e.g., [53]).

Proposition 46 Let $\mu: X \rightarrow T$ be a pure, closed support image, $t \in T$ a label and $(x, y)$ a free pair for the label $t$. If 
any point in $X$ that covers a xel (a minimal point) covers at most one other xel and no other points, then $\mu_{y, t}$ is pure.

Proof Let $\mu: X \rightarrow T$ be a pure, closed support image. We assume that any point in $X$ that covers a xel covers at most one other xel and no other points. Let $(x, y)$ be a free pair for the label $t$. If $\operatorname{ht}(x) \geq 1$ then the xels of $X$ have the same label in $\mu_{y, t}$ as in $\mu$. As $y$ covers $x$ (Proposition 41), if $x$ is a xel, then we derive from the hypothesis that $y^{\downarrow \star} \backslash\{x\}=\emptyset$ or $y^{\downarrow \star} \backslash\{x\}=\{z\}$ for some xel $z \in X$. Then, $\mu_{y, t}(x)=\perp$ or $\mu_{y, t}(x)=\mu(z)$ and $\mu(z)$ is an atom, or $\mu(z)=\perp$, since $\mu$ is pure.

Note that the condition "any point in $X$ that covers a xel (a minimal point) covers at most one other xel and no other points" could be stated in a complex as "any point of height 1 covers at most two xels" but this is generally not equivalent (in a poset, the height of a point that covers a minimal point need not be one). Figure 21 shows some posets, included in $\mathbb{F}^{3}$, for which this condition is, or is not, satisfied.

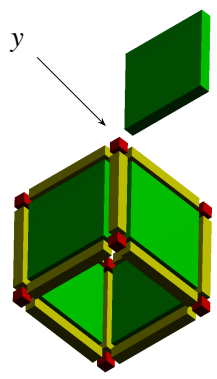

(a)

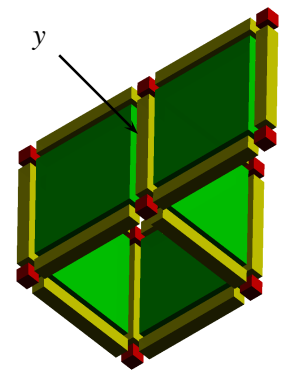

(b)

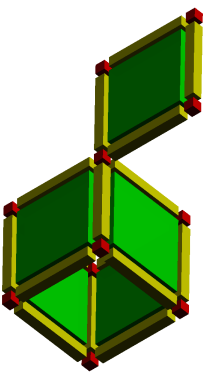

(c)
Fig. 21

(a) A poset (a subset of $\mathbb{F}^{3}$ ) in which a point $y$ covers a xel and covers points that are not xels (1-faces of $\mathbb{F}^{3}$ ). (b) A cubical 2-complex (embedded in $\mathbb{F}^{3}$ ) in which a 1-face $y$ covers three xels. (c) A cubical 2 -complex in which any 1 -face covers at most two xels.

\subsection{Homotopy}

Theorem 47 establishes that connected components and homotopy groups are preserved by cuts provided that the domain of the image has the pierced sphere property (see Subsection 2.8 and the codomain is distributive. Figure 22 illustrates the sequence of changes described in the proof. In Appendix D, some counterexamples show that this preservation is no longer guaranteed when $T$ is not distributive (Figure 30) or when $X$ has not the pierced sphere property (Counterexample64).

Theorem 47 Let $\mu: X \rightarrow T$ be a closed support image and $(x, y)$ a free pair for the label $t \in T$. If $X$ has the pierced

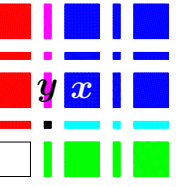

(a)

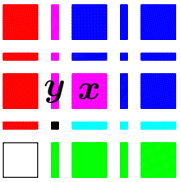

(b)

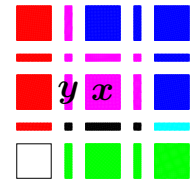

(c)

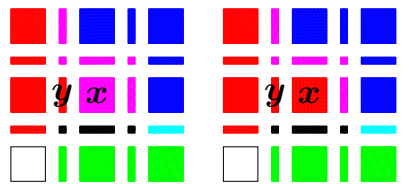

(d) (e)
Fig. 22 The four steps in Theorem 47 (a) The initial closed support image $\mu$ with a free pair $(x, y)$ for the label $\{b\}$ (see Figure 18(b)). (b) The image $\mu+(x, \mu(y))$. (c) The smallest closed support image $v$ greater than or equal to $\mu+(x, \mu(y))$. (d) The image $v+(y,\{r\})$. (e) The image $\mu_{y,\{b\}}=(v+(y,\{r\}))+(x,\{r\})$.

sphere property and if the lattice $T$ is distributive, the cut $\mu_{y, t}$ is equivalent to $\mu$ and, if $X=\mathbb{F}^{n}, \mu_{y, t}$ is strongly equivalent to $\mu$.

Proof 1. By Definition 38 $x$ is simple for the label $\mu(y)$ in the image $\mu$. Thereafter, $\mu^{\prime}=\mu+(x, \mu(y))$ is equivalent to $\mu$ (strongly equivalent if $X=\mathbb{F}^{n}$, according to Proposition 34).

2. Let $v$ be the smallest closed support image greater than or equal to $\mu^{\prime}$. Since $\mu$ is a closed support image, $v$ is defined by $v(z)=\mu(z) \vee \mu(y)$ if $z>x$ and $v(z)=\mu(z)$ otherwise. We shall prove that $v$ is equivalent to $\mu^{\prime}$. To do so, thanks to Proposition 35, it suffices to establish that the points $z \in x^{\uparrow \star}$ with same height $k, k \geq 1$, are simple for the label $v(z)$ in the image $\mu_{k}$ defined by $\mu_{k}(a)=v(a)$ if $a \in x^{\uparrow}$ and $\operatorname{ht}(a)<k$ and $\mu_{k}(a)=\mu(a)$ otherwise. Thereby, according to Definition 31] we consider a point $z$ in $x^{\uparrow \star}$ such that $v(z) \neq \mu(z)$, i.e., $\mu(y) \not \leq \mu(z)$, and let $k$ be the height of $z$. Let $u$ be a label such that $z \in\langle u\rangle_{v}$ and $z \notin\langle u\rangle_{\mu_{k}}$ (if $z \notin\langle u\rangle_{v}$ or $z \in\langle u\rangle_{\mu_{k}}$, then the support of $u$ in the image $\mu_{k}+(z, v(z))$ is equal to the support of $u$ in the image $\left.\mu_{k}\right)$. Observe that $\mu_{k}(z)=\mu(z)$ and $\mu_{k}(x)=\mu(y)$. Then, from $z \in\langle u\rangle_{v}$ and $z \notin\langle u\rangle_{\mu_{k}}$, we derive $\perp \neq v(z) \wedge u=(\mu(z) \vee \mu(y)) \wedge u=\left(\mu_{k}(z) \vee \mu(y)\right) \wedge u=$ $\left(\mu_{k}(z) \vee \mu_{k}(x)\right) \wedge u=\left(\mu_{k}(z) \wedge u\right) \vee\left(\mu_{k}(x) \wedge u\right)=\mu_{k}(x) \wedge u$ (the last equality follows from $z \notin\langle u\rangle_{\mu_{k}}$, whence $\mu_{k}(z) \wedge u=$ $\perp$ ). Thus, $x \in\langle u\rangle_{\mu_{k}}$. As $z \notin\langle u\rangle_{\mu}$ (since $z \notin\langle u\rangle_{\mu_{k}}$ ) and $\mu$ is non-decreasing, no point in $z^{\downarrow}$ is in the support of $u$ in $\mu$. Moreover, as $\mu_{k}=\mu$ on $X \backslash x^{\uparrow}$, no point in $z^{\downarrow} \backslash x^{\uparrow}$ is in the support of $u$ in $\mu_{k}$. Hence, $z^{\downarrow \star} \cap\langle u\rangle_{\mu_{k}}$ has a minimal element, $x$, and is contractible (Property 3 ). Thereby, $z$ is $\beta$-simple for $\langle u\rangle_{\mu_{k}} \cup\{z\}$. This establishes that $z$ is simple for the label $v(z)$ in the image $\mu_{k}$. Thus, the images $v$ and $\mu^{\prime}$ are equivalent, strongly equivalent if $X$ is $\mathbb{F}^{n}$.

3. Let $u=\bigvee_{z \in y^{\downarrow \star} \backslash\{x\}} \mu(z)$. We prove now that $y$ is simple for the label $u$ in the image $v$. Remember that $v(y)=\mu(y)$ 
and $u \leq \mu(y)$ for $\mu$ is non-decreasing. Therefore $w \wedge u \leq$ $w \wedge v(y)$ for all $w \in T$. Let $w$ be a label such that $w \wedge v(y) \neq$ $\perp$ and $w \wedge u=\perp$. Obviously, for all $z \in y^{\downarrow \star} \backslash\{x\}$, we have $\mu(z) \leq u$ and, thereafter, $w \wedge \mu(z) \leq w \wedge u=\perp$. Hence, $y^{\downarrow \star} \cap\langle w\rangle_{v} \subseteq\{x\}$. Now, $x \in\langle w\rangle_{v}$ for $v(x)=\mu(y)=v(y)$. Thus, $y^{\downarrow \star} \wedge\langle w\rangle_{v}=\{x\}$ and $y$ is $\beta$-simple for the support of $w$ in the image $v$. We derive that $y$ is simple for the label $u$ in the image $v$ and that the images $v+(y, u)$ and $v$ are equivalent, strongly equivalent if $X=\mathbb{F}^{n}$.

4. Finally, let us prove that $x$ is simple for the label $u=$ $\bigvee_{z \in y^{\downarrow \star} \backslash\{x\}} \mu(z)$ in the image $v^{\prime}=v+(y, u)$ in which the label of $x$ is $v(x)=\mu(y)$. Remember that we have established that $w \wedge u \leq w \wedge \mu(y)$ for all $w \in T$. Let $w$ be a label such that $w \wedge \mu(y) \neq \perp$ and $w \wedge u=\perp$. Since $v$ is nondecreasing and $x \in\langle w\rangle_{v}$, one has $x^{\uparrow \star} \subseteq\langle w\rangle_{v}$ and thereafter $x^{\uparrow \star} \cap\langle w\rangle_{v^{\prime}}=x^{\uparrow \star} \backslash\{y\}$. Now, by hypothesis, $X$ has the pierced sphere property. Then $x^{\uparrow \star} \backslash\{y\}$ is homotopically trivial and $x$ is a $\gamma$-simple point for $\langle w\rangle_{v^{\prime}}$. Furthermore $x^{\uparrow \star} \cap\langle w\rangle_{v^{\prime}}^{c}=\{y\}$ is clearly contractible so $x$ is a $\beta$-simple point (and thus a $\gamma$-simple point) for $\langle w\rangle_{v^{\prime}}^{c}$. Hence (Property 22), for all labels $w,\langle w\rangle_{v^{\prime}}$ and $\langle w\rangle_{v^{\prime}+(x, u)}$ are weakly homotopy equivalent and $\langle w\rangle_{v^{\prime}}^{c}$ and $\langle w\rangle_{\nu^{\prime}+(x, u)}^{c}$ are weakly homotopy equivalent (if $w$ is such that $w \wedge \mu(y)=\perp$ or $w \wedge u \neq \perp$, the above equivalences are equalities). It is plain that the image $v^{\prime}+(x, u)$ is equal to the cut $\mu_{y, t}$. Thus $\mu_{y, t}$ and $v^{\prime}$ are equivalent (strongly equivalent if $X=\mathbb{F}^{n}$ ). By transitivity, $\mu_{y, t}$ and $\mu$ are equivalent (strongly equivalent if $X=\mathbb{F}^{n}$ ).

\section{Regular label images}

In this section, we are interested in labels images constructed from label digital images, that is, images defined on $\mathbb{Z}^{n}$. The particularity of these label images is that they are entirely determined by their values on the xels (the minimal points of $X$, which are also - by identification- the points of $\mathbb{Z}^{n}$ ).

As $X$ is locally finite, for any point $x \in X$ the set $x^{-}=$ $\left\{y \in x^{\downarrow} \mid \operatorname{ht}(y)=0\right\}$ is not empty and is finite. Thus, we can define the label of a point $x$ in $X$ depending only on the labels of the elements of $x^{-}$.

\subsection{Regular and regularised images}

Definition 48 (Regular label image) A label image $\mu: X \rightarrow$ $T$ is a regular (label) image if, for all $x \in X$,

$\mu(x)=\bigvee_{y \in x^{-}} \mu(y)$

Proposition 49 Let $\mu: X \rightarrow T$ be a regular label image. Then, $\mu$ is a closed support image.
Proof It is plain that, for any point $x, y$ in $X, x \leq y \Rightarrow x^{-} \subseteq$ $y^{-} \Rightarrow \mu(x) \leq \mu(y)$. Hence, a regular label image is nondecreasing and thereafter is a closed support image (Proposition 36).

The regularisation of a label image $\mu$ is the regular image $\mu^{\prime}$ which coincides with $\mu$ on the xels of $X$.

If $\mu$ is a closed support image and $\mu^{\prime}$ is its regularisation, then $\mu^{\prime}(x)=\bigvee_{y \in x^{-}} \mu^{\prime}(y)=\bigvee_{y \in x^{-}} \mu(y) \leq \mu(x)$ (for $\mu$ is non-decreasing) for all $x \in X$. It can easily be seen that the regularisation of a closed support image is the smallest closed support image which coincides with $\mu$ on the xels of X.

Let us define the function $\zeta: L^{\mathbb{Z}^{n}} \rightarrow T^{X}$ which maps, in a one-to-one manner, a label digital image on a regular image. Given a label digital image $\lambda: \mathbb{Z}^{n} \rightarrow L, \zeta(\lambda): X \rightarrow$ $T$ is the only regular image such that, for any xel $x \in X$, $\zeta(\lambda)(x)=\lambda(x)$ (actually, $\zeta(\lambda)(i(x))=j(\lambda(x))$ where $i$ and $j$ are respectively the embedding of $\mathbb{Z}^{n}$ in $X$ and of $L$ in $T$ ).

In general, the binary images $\mu \wedge l$, where $\mu$ is a regular image and $l \in L$ is a proto-label, are not regular (see Counterexample 65 in Appendix (D). Nevertheless, if we regularise these binary images, we find that any regular image is a supremum of regular binary images.

Proposition 50 Let $\mu: X \rightarrow T$ be a regular label image. Let $\left\{l_{i}\right\}_{i=1}^{\ell}(\ell \geq 1)$ be the set of the atoms of $T$. Then $\mu=\bigvee_{i=1}^{\ell} \mu_{i}^{\prime}$ where, for all $i \in[1, \ell], \mu_{i}^{\prime}$ denotes the regularisation of the binary image $\mu_{i}=\mu \wedge l_{i}$.

Proof We prove first that a supremum of regular images is regular:

$$
\begin{aligned}
\left(\bigvee_{i=1}^{l} \mu_{i}^{\prime}\right)(x)=\bigvee_{i=1}^{\ell} \mu_{i}^{\prime}(x) & =\bigvee_{i=1}^{\ell} \bigvee_{y \in x^{-}} \mu_{i}^{\prime}(y) \\
& =\bigvee_{y \in x^{-}}^{\ell} \bigvee_{i=1}^{\ell} \mu_{i}^{\prime}(y)=\bigvee_{y \in x^{-}}\left(\bigvee_{i=1}^{l} \mu_{i}^{\prime}\right)(y) .
\end{aligned}
$$

Now, obviously, $\mu_{i}^{\prime}(y)=\mu_{i}(y)$ for all xel $y$ and therefore, $\bigvee_{i=1}^{\ell} \mu_{i}^{\prime}(y)=\bigvee_{i=1}^{\ell} \mu_{i}(y)$ for all xel $y$. From Proposition 30 , we have $\mu=\bigvee_{i=1}^{\ell} \mu_{i}$. Thus, $\mu$ and $\bigvee_{i=1}^{\ell} \mu_{i}^{\prime}$ are regular images which coincide on the xels of $X$. Hence, $\mu=\bigvee_{i=1}^{\ell} \mu_{i}^{\prime}$.

\subsection{Regular images onto a Boolean lattice}

In this subsection, we assume the lattice $T$ to be Boolean. For all pair $(t, u)$ of labels, we set $t \backslash u=t \wedge u^{c}$ where $u^{c}$ is the complement of $u$ in $T$.

The next proposition establishes that the reduction of the number of labels, by identification of some labels with the background, preserves the regularity of the image. 
Proposition 51 Let $\mu: X \rightarrow T$ be a regular image. Let $t \in T$ be a label. Then, the image $\mu \wedge t: X \rightarrow t^{\downarrow}$ defined, for all $x \in X$, by $(\mu \wedge t)(x)=\mu(x) \wedge t$, is regular.

Proof For any point $x \in X$, we have $(\mu \wedge t)(x)=\mu(x) \wedge$ $t=\left(\bigvee_{y \in x^{-}} \mu(y)\right) \wedge t=\bigvee_{y \in x^{-}}(\mu(y) \wedge t)=\bigvee_{y \in x^{-}}(\mu \wedge t)(y)$. Therefore, the image $\mu \wedge t$ is regular.

Applied to proto-labels $l_{i}$, Proposition 51 says that the binary images $\mu \wedge l_{i}$, whose supremum is $\mu$ (see Proposition 30), are regular.

With the following proposition, we show that the function $\zeta$ permutes with the reduction of the lattice $T$ to $t^{\downarrow}$ for any label $t \in T$.

Proposition 52 Let $\lambda: \mathbb{Z}^{n} \rightarrow$ L be a label digital image. Then 9 for all $t \in T, \zeta(\lambda) \wedge t=\zeta(\lambda \wedge t)$.

In other words, we have the following commutative diagram:

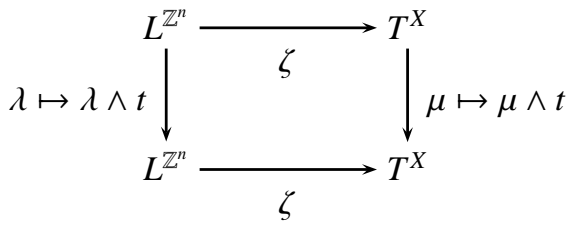

Proof Since the images $\zeta(\lambda) \wedge t$ and $\zeta(\lambda \wedge t)$ are regular (from Proposition 51 and the very definition of $\zeta$ ), it suffices to show that they are equal on the xels of $X$. Let $x$ be a xel. On one side, one has $\zeta(\lambda \wedge t)(x)=(\lambda \wedge t)(x)=\lambda(x) \wedge t$ and on the other side, $(\zeta(\lambda) \wedge t)(x)=\zeta(\lambda)(x) \wedge t=\lambda(x) \wedge t$. Thus, $\zeta(\lambda \wedge t)(x)=(\zeta(\lambda) \wedge t)(x)$.

After reducing the number of labels by taking the infimum with a particular label $t$, we can consider the remaining labels as a unique label. The result is a binary image whose support is $\langle t\rangle$. Starting from a label digital image, the following proposition shows that this operation can be made before or after the use of the function $\zeta$. Combining this proposition with Proposition 52 and the results established in [25], it means that the connected components and the digital fundamental groups of any binary digital image obtained by just considering a particular union of labels in a label digital image are isomorphic to the ones obtained by the same operation in the label image.

In Proposition 53, the lattice $T$ need not be distributive.

Proposition 53 Let $\lambda: \mathbb{Z}^{n} \rightarrow$ L be a label digital image. Let $B . \lambda: \mathbb{Z}^{n} \rightarrow\{\perp, \top\}$ be the binary image defined by $B . \lambda(z)=$ $\perp$ if $\lambda(z)=\perp$ and $B . \lambda(z)=\top$ otherwise. Let $B . \zeta(\lambda)$ : $X \rightarrow\{\perp, \top\}$ be the binary image defined by $B . \zeta(\lambda)(z)=\perp$ if $\zeta(\lambda)(z)=\perp$ and $B . \zeta(\lambda)(z)=\top$ otherwise. Then, $B . \zeta(\lambda)=$ $\zeta(B . \lambda)$

9 The notation is a bit tricky here. In fact, since $t \notin L$, we should define $\lambda \wedge t$ by $(\lambda \wedge t)(z)=\lambda(z)$ if $\lambda(z)$ is an atom under $t$ and $(\lambda \wedge t)(z)=\perp$ otherwise. Of course, we have $\zeta(\lambda)(z) \wedge t=(\lambda \wedge t)(z)$ for $\lambda(z)$ is an atom.

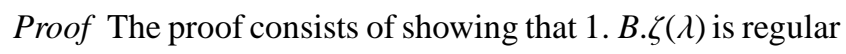
and 2. the functions $B . \zeta(\lambda)$ and $\zeta(B . \lambda)$ coincide on the xels of $X$.

1. Let $\mu: X \rightarrow T$ be a regular image, $B . \mu: X \rightarrow\{\perp, \top\}$ be the binary image defined by $B . \mu(z)=\perp$ if $\mu(z)=\perp$ and $B . \mu(z)=\top$ otherwise and $x$ be a point of height greater than or equal to 1 . We have: $B . \mu(x)=\perp \Leftrightarrow \mu(x)=$ $\perp \Leftrightarrow \bigvee_{y \in x^{-}} \mu(y)=\perp \Leftrightarrow \forall y \in x^{-}, \mu(y)=\perp \Leftrightarrow \forall y \in$ $x^{-}, B . \mu(y)=\perp \Leftrightarrow \bigvee_{y \in x^{-}} B . \mu(y)=\perp$. We can straightforwardly conclude that $B . \mu$ is regular.

2. Let $x$ be a xel. We have: $B . \zeta(\lambda)(x)=\perp \Leftrightarrow \zeta(\lambda)(x)=$ $\perp \Leftrightarrow \lambda(x)=\perp \Leftrightarrow B . \lambda(x)=\perp \Leftrightarrow \zeta(B . \lambda)(x)=\perp$. Hence, $B . \zeta(\lambda)=\zeta(B . \lambda)$ are equal on the xels of $X$ and, since they are regular, they are equal.

The following lemma gives a way to locally regularise some closed support images. We will use this lemma in Subsection 5.3 to regularise a label image after a cut.

Lemma 54 Let $\mu: X \rightarrow T$ be a closed support image and $\mu^{\prime}$ be the regularisation of $\mu$. Let $(x, y)$ be a free pair for the label $t=\mu(x) \backslash \mu^{\prime}(x)$ in the image $\mu$ such that $\mu(x)=\mu(y)$. Then, the cut $\mu_{y, t}$ is equal to $\mu$ on $X \backslash\{x, y\}$ and to $\mu^{\prime}$ on $\{x, y\}$.

Proof Since $\mu(x)=\mu(y)$ and $\mu$ is non-decreasing, $\mu(y) \leq$ $\mu(z)$ for all $z \in x^{\uparrow}$. Now, for any point $z \in x^{\uparrow \star} \backslash\{y\}$, by Definition 42 $\mu_{y, t}(z)=\mu(z) \vee \mu(y)$ and thereafter, $\mu_{y, t}(z)=\mu(z)$. By Definition 42 again, $\mu_{y, t}(z)=\mu(z)$ for any point $z$ in $X \backslash x^{\uparrow}$. Thus, $\mu_{y, t}$ is equal to $\mu$ on $X \backslash\{x, y\}$.

As $(x, y)$ is a free pair for $t$, we derive that $t \neq \perp$. In particular, $x$ is not a xel (by definition, $\mu^{\prime}$ coincides with $\mu$ on the xels of $x$ ). Then:

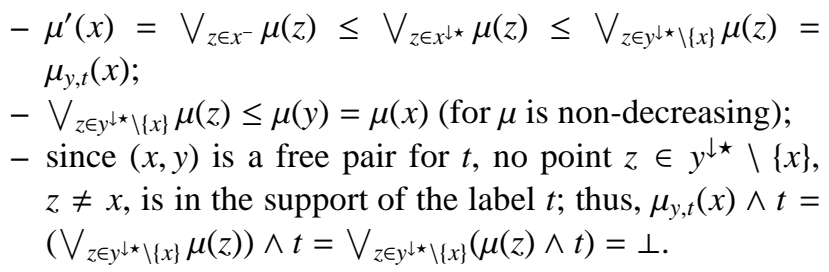

The lattice $T$ is distributive, so it is modular (see Appendix A). Then, since $\mu^{\prime}(x) \leq \mu_{y, t}(x) \leq \mu(x)$ and $\mu_{y, t}(x) \wedge t=\perp$, we get: $\mu_{y, t}(x)=\mu_{y, t}(x) \wedge \mu(x)=\mu_{y, t}(x) \wedge\left(t \vee \mu^{\prime}(x)\right)=$ $\left(\mu_{y, t}(x) \wedge t\right) \vee \mu^{\prime}(x)=\mu^{\prime}(x)$.

As regards the point $y$, we have $\mu_{y, t}(y)=\mu_{y, t}(x)=\mu^{\prime}(x) \leq$ $\mu^{\prime}(y)=\bigvee_{z \in y^{-}} \mu(z) \leq \bigvee_{z \in y \downarrow \star \backslash\{x\}} \mu(z)=\mu_{y, t}(y)$. Hence, $\mu_{y, t}(y)=$ $\mu^{\prime}(y)$.

\subsection{Digitally simple xels}

A cut in a regular image is seldom regular. For instance, the cut of Figure 19(b) is not regular since the 1-face under $x$ is 


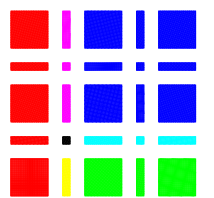

(a)

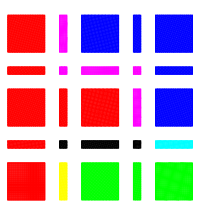

(b)

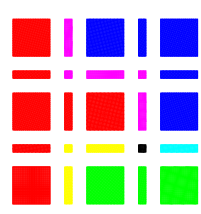

(c)

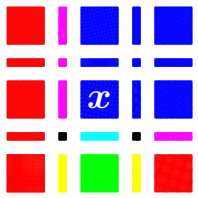

(a)

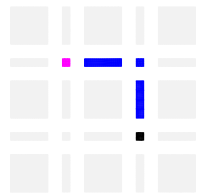

(b)

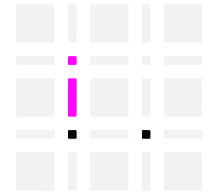

(c)
Fig. 24 (a) A regular image $\mu: \mathbb{F}^{2} \rightarrow 2^{\{r, g, b\}}$. (b) The set $\operatorname{Att}(x,\{b\})$. (c) The set $\operatorname{Att}(x,\{r\})$.

We set also $\operatorname{Card}(t)=\operatorname{Card}\left(\left\{u \in L^{\star} \mid u \leq t\right\}\right)=\operatorname{Card}\left(t^{\downarrow} \cap\right.$ $\left.L^{\star}\right)$. The integer $\operatorname{Card}(t)$ is the number of proto-labels under the label $t$.

The following proposition provides a sufficient condition for a xel $x$ to be digitally simple for a label $t \in T$ in a pure and regular image $\mu$. It is required the existence of a free pair $(x, y)$ for the label $\mu(x)$ with $y \in\langle t\rangle$ (condition $(i)$ ), the possibility to shrink $x^{\uparrow}$ onto $\operatorname{Att}(x, \mu(x))$ by withdrawal of (combinatorial) free pairs in such a way that the points whose label is less than or equal to the label of $y$ are removed first (condition (ii)) and that no point in $x^{\uparrow} \backslash \operatorname{Att}(x, \mu(x))$ has more than one proto-label distinct from those of $y$ (condition (iii)). The proof consists of regularising step by step (thanks to Lemma 54) the labels of the points of $x^{\uparrow} \backslash\{x, y\}$ in the nonregular image $\mu_{y, \mu(x)}$, beginning by the points whose label is less than or equal to the one of $y$. Figure 25 illustrates some of these steps. In Appendix D, Counterexample 66 shows that in the following proposition, condition (iii) is not necessary. This condition is used in the second part of the proof to ensure that for any free pair considered, the two points share the same label. Thereby, our example is built in such a way that this last property is true, even if condition (iii) is not respected.

Proposition 56 Let $\mu: X \rightarrow T$ be a pure and regular image whose codomain $T$ is distributive and whose domain $X$ is such that any point in $X$ that cover a xel covers at most one other xel and no other points. Let $t$ be a label of $T$ and $x a$ xel of $X$, not in $\langle t\rangle$. If:

(i) there exists a point $y \in\langle t\rangle$ such that $(x, y)$ is a free pair for the label $\mu(x)$, simple for $t$ if there exists a sequence of cuts $\left(\mu_{i}\right)_{i=0}^{r}, r \geq 0$, where $\mu_{0}=\mu, \mu_{i}$ is a cut in $\mu_{i-1}$ for all $i \in[1, r], \mu_{r}$ is regular, $x \in\langle t\rangle_{\mu_{r}}$ and $\mu(y)=\mu_{r}(y)$ for any xel $y$ distinct from $x$.

In the sequel, so we do not impose the space $X$ to be $\mathbb{F}^{n}$, we borrow the notion of attachment to authors that have worked on image processing in the framework of cubical complexes [54,23].

Let $\mu: X \rightarrow T$ be a regular image, $x$ a xel in $X$ and $t$ a label in $T$. We set $\operatorname{Att}(x, t)=x^{\uparrow \star} \cap\langle t\rangle_{\mu^{\prime}}$ where the image $\mu^{\prime}$ is the regularisation of $\mu+(x, \perp)$. The points in $\operatorname{Att}(x, t)$ are the points that "attach" the xel $x$ to the support of $t$ (see Figure 24). (ii) $x^{\uparrow} \backslash\{x, y\} \backslash\left(x^{\uparrow} \backslash \mu^{-1}\left(\mu(y)^{\downarrow}\right)\right) \cup A \searrow A$,

(iii) for any point $z \in x^{\uparrow \star} \backslash A$, $\operatorname{Card}(\mu(z) \backslash \mu(y)) \leq 1$,

where $A=\operatorname{Att}(x, \mu(x)))$, then the xel $x$ is digitally simple for the label $t$.

Proof We set $t_{0}=\mu(x)$. Since $(x, y)$ is a free pair for the label $t_{0}$, it is also a combinatorial free pair for the set $\left\langle t_{0}\right\rangle$. Let $\left(\left(x_{i}, y_{i}\right)\right)_{i=0}^{r}$ be a sequence of combinatorial free pairs from $x^{\uparrow}$ to $A=\operatorname{Att}\left(x, t_{0}\right)$ such that $x_{0}=x, y_{0}=y$ and $\bigcup_{i=0}^{k}\left\{x_{i}, y_{i}\right\}=$ $\left(x^{\uparrow} \cap \mu^{-1}\left(\mu(y)^{\downarrow}\right)\right) \backslash A$ with $k \in[0, r]$. We set $t_{1}=\mu(y) \backslash t_{0}$. From the hypothesis on $X$ and $\mu$, we derive that $t_{1}$ is an atom and, 


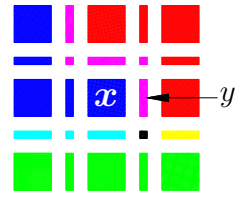

(a)

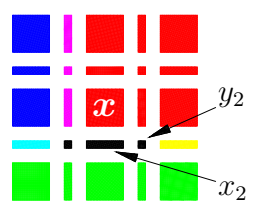

(c)

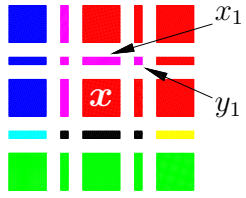

(b)

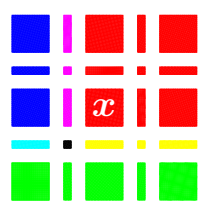

(d)
Fig. 25 (a) The label image $\mu$ and $(x, y)=\left(x_{0}, y_{0}\right)$, a free pair for $\mu(x)$. (b) The label image $\mu_{1}$ and $\left(x_{1}, y_{1}\right)$, a combinatorial free pair for the set $x^{\uparrow} \backslash\{x, y\}$ whose faces are not in $\operatorname{Att}(x, \mu(x))$ and whose labels are less than or equal to $\mu(y)$. (c) The label image $\mu_{2}=\mu_{k+1}$ and $\left(x_{2}, y_{2}\right)=$ $\left(x_{k+1}, y_{k+1}\right)$, a combinatorial free pair for the set $x^{\uparrow} \backslash \bigcup_{j=0}^{k}\left\{x_{j}, y_{j}\right\}$ whose faces are not in $\operatorname{Att}(x, \mu(x))$. (d) The label image $\mu^{\prime}=\mu_{r}$.

thereafter, that $t_{1} \leq t$. Let $\mu_{1}$ be the cut $\mu_{y, t_{0}}$. By Definition 42. $\mu_{1}(h)=t_{1}$ if $h \in\{x, y\}, \mu_{1}(h)=\mu(h) \vee t_{1}$ if $h \in x^{\uparrow}$ । $\{x, y\}$ and $\mu_{1}(h)=\mu(h)$ otherwise. In particular, $t_{0} \vee t_{1} \leq$ $\mu_{1}(h)$ for any point $h \in x^{\uparrow} \backslash\{x, y\}$ (for $\mu$ is non-decreasing). By the very definition of $k$, for any point $h \in \bigcup_{i=0}^{k}\left\{x_{i}, y_{i}\right\}$, $\mu_{1}(h)=\mu(h) \vee t_{1} \leq t_{0} \vee t_{1}$. Therefore, $\mu_{1}(h)=t_{0} \vee t_{1}$ for any point $h \in \bigcup_{i=1}^{k}\left\{x_{i}, y_{i}\right\}$. In particular, $\mu_{1}\left(x_{1}\right)=\mu_{1}\left(y_{1}\right)$. Observe that, since $\mu$ is regular, $\mu_{1}=\mu^{\prime} \vee \nu_{1}$ where $\mu^{\prime}$ is the regularisation of $\mu_{1}$ and $\nu_{1}(h)=t_{0}$ if $h \in x^{\uparrow} \backslash(\{x, y\} \cup A)$ and $v_{1}(h)=\perp$ otherwise $\left(v_{1}=\mu_{1} \backslash \mu^{\prime}\right)$. Now $x_{1} \in\left\langle t_{0}\right\rangle_{\mu_{1}}$ and $\left(\left\langle t_{0}\right\rangle_{\mu_{1}} \cap y_{1}^{\downarrow}\right) \backslash x^{\uparrow}=\left(\left\langle t_{0}\right\rangle_{\mu} \cap y_{1}^{\downarrow}\right) \backslash x^{\uparrow}$ is empty for $y_{1} \notin$ A. Thus, $\left(x_{1}, y_{1}\right)$, which is a combinatorial free pair in $x^{\uparrow} \backslash$ $\{x, y\}$, is also a combinatorial free pair in $\left\langle t_{0}\right\rangle_{\mu_{1}}$. Then, from Proposition 40, $\left\{x_{1}, y_{1}\right\}$ is a free pair for the label $t_{0}=v_{1}\left(x_{1}\right)$. The cut $\mu_{2}=\left(\mu_{1}\right)_{y_{1}, t_{0}}$ verifies $\mu_{2}(h)=\mu^{\prime}(h)$ if $h \in\left\{x_{1}, y_{1}\right\}$ and $\mu_{2}(h)=\mu_{1}(h)$ otherwise (Lemma 54). Thereby, gradually, we can show that the pairs $\left(x_{i}, y_{i}\right), 1 \leq i \leq k$, are free for $t_{0}$ in the image $\mu_{i}=\mu^{\prime} \vee v_{i}$ where $v_{i}(h)=t_{0}$ for all $h \in$ $x^{\uparrow} \backslash\left(A \cup \bigcup_{j=0}^{i-1}\left\{x_{j}, y_{j}\right\}\right)$ and $v_{i}(h)=\perp$ otherwise.

The pair $\left(x_{k+1}, y_{k+1}\right)$ is in $x^{\uparrow} \backslash \mu^{-1}\left(\left\{t_{0}, t_{0} \vee t_{1}\right\}\right)$ thus we have $t_{0} \vee t_{1}<\mu_{k}\left(x_{k+1}\right)=\mu\left(x_{k+1}\right) \vee t_{1}$ and $t_{0} \vee t_{1}<\mu_{k}\left(y_{k+1}\right)=$ $\mu\left(y_{k+1}\right) \vee t_{1}$. Now, $\operatorname{Card}\left(\mu\left(x_{k+1}\right) \backslash\left(t_{0} \vee t_{1}\right)\right)=\operatorname{Card}\left(\mu\left(y_{k+1}\right) \backslash\right.$ $\left.\left(t_{0} \vee t_{1}\right)\right) \leq 1$ (Hypothesis (iii)). Hence, necessarily, we have $\operatorname{Card}\left(\mu\left(x_{k+1}\right) \backslash\left(t_{0} \vee t_{1}\right)\right)=\operatorname{Card}\left(\mu\left(y_{k+1}\right) \backslash\left(t_{0} \vee t_{1}\right)\right)=1$. Since $\mu_{k}\left(x_{k+1}\right) \leq \mu_{k}\left(y_{k+1}\right)$, for $\mu$ is a closed support image and cuts of closed support images are closed support images (Proposition 45), we have $\mu_{k}\left(x_{k+1}\right)=\mu_{k}\left(y_{k+1}\right)$. Thereafter we deduce as above that $\left(x_{k+1}, y_{k+1}\right)$ is a free pair in $\mu_{k}$ for $t_{0}$ and the cut $\mu_{k+1}$ is equal to $\mu^{\prime} \vee v_{k+1}$ with $v_{k+1}(h)=t_{0}$ for all $h \in x^{\uparrow} \backslash\left(A \cup \bigcup_{j=0}^{k}\left\{x_{j}, y_{j}\right\}\right)$ and $v_{i}(h)=\perp$ otherwise. We continue the same reasoning on each pair $\left(x_{i}, y_{i}\right)$ for $k+2 \leq$ $i \leq r$. The last cut is $\mu_{r}$ with $\mu_{r}=\mu^{\prime} \vee v_{r}$ where $v_{r}(h)=t_{0}$ for all $h \in x^{\uparrow} \backslash\left(A \cup \bigcup_{j=0}^{r}\left\{x_{j}, y_{j}\right\}\right)$ and $v_{r}(h)=\perp$ otherwise, i.e., $v_{r}=\perp$ and $\mu_{r}=\mu^{\prime}$. So, we are done.
In [55], Couprie and Bertrand have established a "confluence" property for collapses inside a cubical cell of dimension 2, 3 or 4: if $x^{\uparrow} \searrow \operatorname{Att}(x,\langle t\rangle)$ and $X$ is a complex such that $\operatorname{Att}(x,\langle t\rangle) \subset X \subset x^{\uparrow}$, then $x^{\uparrow} \searrow X$ iff $X \searrow$ $\operatorname{Att}(x,\langle t\rangle)$. Thanks to this property, we can apply Proposition 56 to test whether a xel $x \in \mathbb{F}^{n}(n \leq 4)$ is digitally simple for a label $t$ by the mean of the following greedy algorithm. Of course if the following algorithm returns "false", it just means that the hypothesis of Proposition 56 are not all satisfied and, since this proposition only provides sufficient conditions, the tested xel can nevertheless be digitally simple. Figure 26 provides examples of images obtained from the same label digital image by applying the following algorithm to perform thinning or growing on the support of a label.

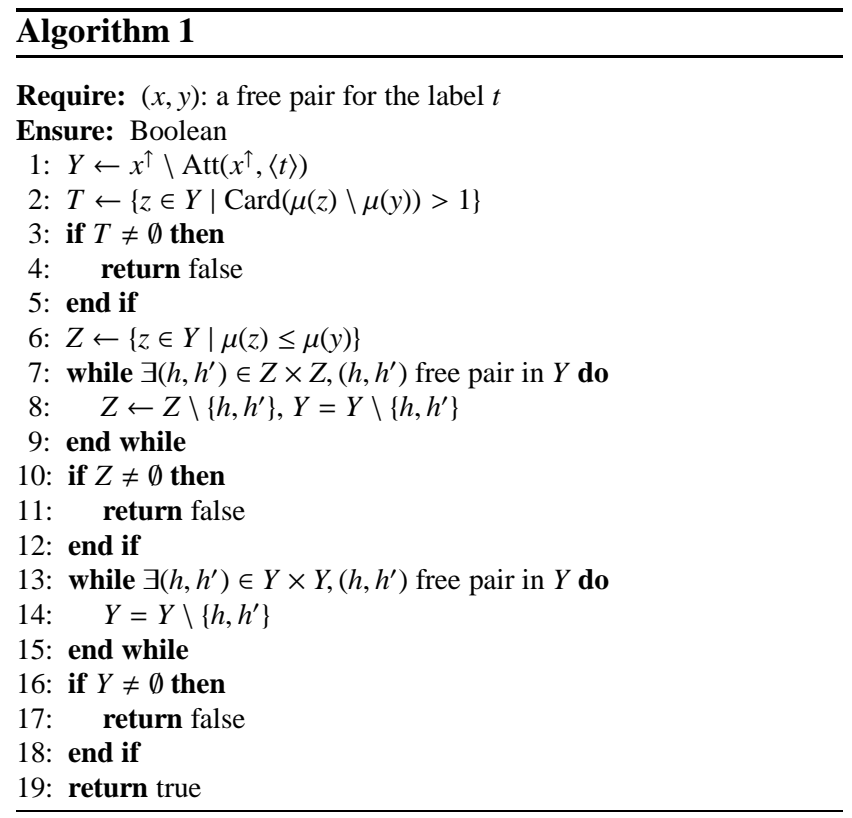

\section{Conclusion}

In this article we have proposed some tools to locally modify a label image with respect not only to the topologies of the labels but also to the topology of the partition, in the sense that the topologies of any union of labels can also be preserved (depending on the choice for the lattice of labels). Here, topology preservation is understood as the existence of weak homotopy equivalence: when a point $x$ is removed from a set $X$, the inclusion $i: X \backslash\{x\} \rightarrow X$ puts in one-to-one correspondence the connected components of $X \backslash\{x\}$ and $X$ and induces isomorphisms between the homotopy groups of both spaces.

Let us now have a look at some of the more relevant models for label images evoked in the introduction. Assum- 


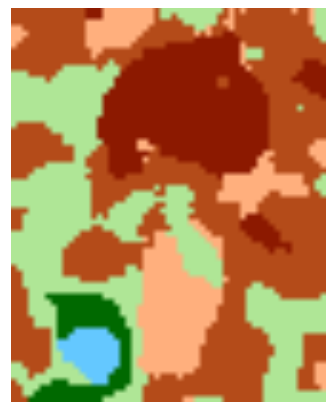

(a)

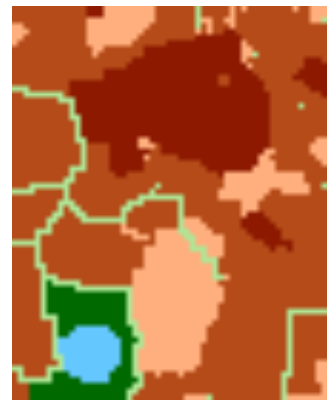

(c)

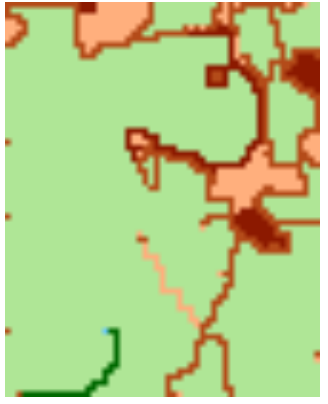

(e)

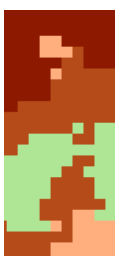

(g)

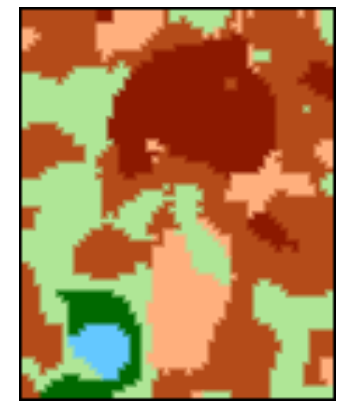

(b)

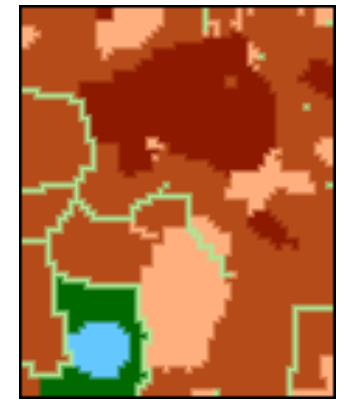

(d)

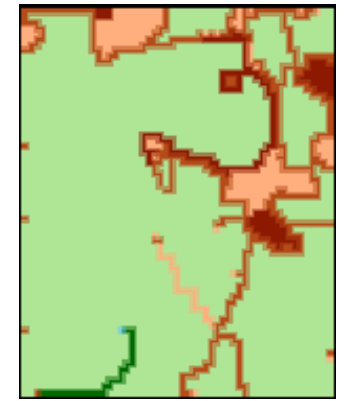

(f)

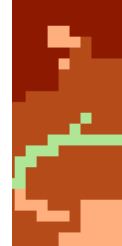

(h)

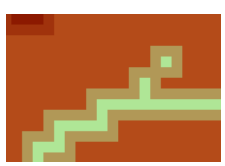

(j)

Fig. 26 (a) A label digital image $\lambda_{0}: \mathbb{Z}^{2} \rightarrow L$ (the background is not depicted). (b) The regular image $\mu=\zeta\left(\lambda_{0}\right): \mathbb{F}^{2} \rightarrow 2^{L^{\star}}$. (c) The preimage $\lambda_{1}=\zeta^{-1}\left(\mu_{1}\right)$ where $\mu_{1}$ is obtained from $\mu$ by applying Algorithm 1 to shrink the green label. (d) The regular image $\mu_{1}$. (e) The pre-image $\lambda_{2}=\zeta^{-1}\left(\mu_{2}\right)$ where $\mu_{2}$ is obtained from $\mu$ by applying Algorithm 1 to expand the green label. (f) The regular image $\mu_{2}$. (g-i) The same detail in the images $\lambda_{0}, \lambda_{1}, \lambda_{2}$. (j) A part of the above detail in the image $\mu_{1}$. Observe that the isolated green square is not digitally simple for the brown label: the change of label will fill a hole in the brown label. ing $X=\mathbb{F}^{n}$, it can be seen that we can process 3D well composed images inside our framework in such a way that the result is still a well composed image by adding a condition in Algorithm 1, any proto-label on a 1-face of the xel being processed must be present on at least one of the two 2-faces including the 1-face and included in the xel (in 2D, no condition is required). The requirement found in [20] to preserve the topology of any union of two or three labels is obviously satisfied in our model since we preserve the topology of any union of labels when $T$ is the power set of the proto-labels. Moreover, we observe that Algorithm 1 forbids to have more than three labels in the neighbourhood of a point adjacent to the processed xel (but it is a point of $X$, not a point of $\mathbb{Z}^{3}$ ). In [15], the authors provide eight figures, five in $2 \mathrm{D}$ and three in 3D, to illustrate their definition of simple points in label images. On two of them, all conditions are satisfied and the point is actually simple. On the six other figures, at least one condition is not satisfied and the point is not simple. We have tested our own conditions on this set of examples and we have obtained the same conclusions (see Appendix E). Thereby it seems that we are able to encompass several approaches with the model presented in this article, providing a framework to write precise topological statements and establish reliable proofs.

Nevertheless, some questions remain. Can Theorem 27 be extended to a wider family of spaces? This would ensure strong equivalences between label images in other spaces than $\mathbb{F}^{n}$. Is it possible to enrich the model in order to be able to work with other types of regular images as those defined in [22]? This could be interesting for the modelling of the $(18,6)$-adjacency pair in $\mathbb{Z}^{3}$. We hope to be able to give some answers to these issues in further works.

\section{A Lattices}

In this appendix, we recall some vocabulary and properties used in the article. More information on lattices can be found in, e.g., [56] or [57].

Lattice. A lattice is a poset in which every pair $(a, b)$ of elements have a supremum, denoted $a \vee b$, and an infimum, denoted $a \wedge b$. Thereafter in a finite lattice, there exists a least and a greatest element.

Atom/Atomistic. In a lattice, an element is an atom if it covers the minimal element. An atomistic lattice is a lattice in which each element that is not the least element is a supremum of a set of atoms.

Modular. A lattice is modular if $x \leq z$ implies $x \vee(y \wedge z)=(x \vee y) \wedge z$.

Distributive. A lattice is distributive if $x \vee(y \wedge z)=(x \vee y) \wedge(x \vee z)$, or, equivalently, if $x \wedge(y \vee z)=(x \wedge y) \vee(x \wedge z)$. A distributive lattice is modular.

Boolean. In a lattice, $y$ is a complement of $x$ if the infimum of $x$ and $y$ is the minimum element and the supremum of $x$ and $y$ is the maximum element. A lattice is Boolean if it is distributive and each element has a complement. Moreover, in this case, this complement is unique. A finite distributive lattice is Boolean iff it is atomistic.

Opening. Let $L$ be a lattice. A function $\varphi: L \rightarrow L$ is an opening if $\varphi$ is anti-extensive $(\varphi(x) \leq x$ for all $x \in L)$ and $\varphi(x) \leq y \Rightarrow \varphi(x) \leq \varphi(y)$ for all $x, y \in L$. An opening is order-preserving $(x \leq y \Rightarrow \varphi(x) \leq$ $\varphi(y))$ and idempotent $(\varphi \circ \varphi=\varphi)$. Let $A$ be a subset of $L$. The 
function $\varphi_{A}: L \rightarrow L$ defined by $\varphi_{A}(x)=\bigvee\{a \in A \mid a \leq x\}$ is an opening.

\section{B Proof of Proposition 26}

The proof of Proposition 26 (Subsection 2.8) relies on some combinatorial properties of cubical and simplicial complexes that we establish hereafter.

Lemma 57 Let $X$ be a cubical or a simplicial complex equipped with the order $\supseteq$. Let $x \in X$ be an $m$-face $(0 \leq m \leq \operatorname{dim}(X))$.

(i) Let $y \in x^{\uparrow}$ be a $k$-face $(0 \leq k \leq m)$. There exist exactly $m-k$ faces in $x^{\uparrow}$ of dimension $(k+1)$ which include $y$.

(ii) Let $x_{1}, x_{2}$ be two faces in $x^{\uparrow}$ such that $\operatorname{dim}\left(x_{1}\right)=m-1, x=$ $x_{1} \sqcup x_{2}\left[10\right.$. Let $Z$ be the set of faces in $x^{\uparrow}$ that intersect both $x_{1}$ and $x_{2}$. The function $\theta: Z \rightarrow x_{1}^{\uparrow}$ defined by $\theta(z)=z \cap x_{1}$ is a bijection and $\operatorname{dim}(\theta(z))=\operatorname{dim}(z)-1$ for all $z \in Z$.

Proof (i) If $k=m$, Lemma[57] is trivial. We suppose now that $k<m$. If $X$ is a simplicial complex, there are $m+1$ vertices in $x$ and $k+1$ vertices in $y$. Hence, there exist exactly $(m+1)-(k+1)=m-k$ faces of $x$ of dimension $k+1$ including $y$ (thus containing the $k+1$ vertices of $y$ plus one). If $X$ is a cubical $n$-complex, we can assume without loss of generality that $x=\prod_{i=1}^{n} I_{i}$ where $I_{i} \in \mathbb{F}_{1}^{1}$ if $i \leq m, I_{i} \in \mathbb{F}_{0}^{1}$ otherwise (see Subsection 2.2) and $y=\prod_{i=1}^{n} J_{i}$ where $\emptyset \subset J_{i} \subset I_{i}$ if $i \leq m-k$ and $J_{i}=I_{i}$ otherwise. It is plain that the only $(k+1)$-faces included in $x$ and including $y$ are the $m-k$ faces $z_{j}, 1 \leq j \leq m-k$ defined by $z_{j}=\prod_{i=1}^{n} K_{i}$ with $K_{i}=J_{i}$ if $i \neq j$ and $K_{j}=I_{j}$.

(ii) If $X$ is a simplicial complex, because $\operatorname{dim}\left(x_{1}\right)=\operatorname{dim}(x)-1$ and $x=x_{1} \sqcup x_{2}, x_{2}$ is a singleton. Then, for all $z \in Z, \theta(z)=z \cap x_{1}=$ $z \backslash x_{2}$. So, it is plain that $\theta$ is a bijection whose inverse $\theta^{-1}$ is defined by $\theta^{-1}(z)=z \cup x_{2}$. Furthermore, for all $z \in Z, \operatorname{dim}(z)>0$ and the simplex $z \cap x_{1}=z \backslash x_{2}$ has dimension $k-1$.

If $X$ is a cubical complex, because $x=x_{1} \sqcup x_{2}$, we have $\operatorname{dim}\left(x_{2}\right)=$ $\operatorname{dim}\left(x_{1}\right)=m-1$. As above, we can assume that $x=\prod_{i=1}^{n} I_{i}$ where $I_{i} \in \mathbb{F}_{1}^{1}$ if $i \leq m, I_{i} \in \mathbb{F}_{0}^{1}$ otherwise, $x_{1}=\prod_{i=1}^{n} J_{i}^{1}$ and $x_{2}=\prod_{i=1}^{n} J_{i}^{2}$ with $J_{i}^{1}=J_{i}^{2}=I_{i}$ if $i \neq m, \emptyset \subset J_{m}^{1} \subset I_{m}$ and $J_{m}^{2}=I_{m} \backslash J_{m}^{1}$. In these conditions, it can easily be seen that $Z=\left\{\prod_{i=1}^{n} K_{i} \mid K_{i}=I_{i}\right.$ if $i \geq$ $m$, and $\emptyset \subset K_{i} \subseteq I_{i}$, otherwise $\}$ and $\theta\left(\prod_{i=1}^{n} K_{i}\right)=\prod_{i=1}^{n} K_{i}^{\prime}$ with $K_{m}^{\prime}=J_{m}^{1}$ and $K_{i}^{\prime}=K_{i}$ otherwise. Hence, $\theta$ is bijective. Moreover, obviously, $\operatorname{Card}\left(\left\{i \mid K_{i}^{\prime} \in \mathbb{F}_{1}^{1}\right\}\right)=\operatorname{Card}\left(\left\{i \mid K_{i} \in \mathbb{F}_{1}^{1}\right\}\right)-1$.

We establish below a proposition which straightforwardly provides Proposition 26 as a corollary. This proposition will be used in the proof of Theorem 27. (see Appendix C). Some steps of the proof are depicted in Figure 27

Proposition 58 Let $X$ be a cubical or a simplicial complex equipped with the order $\supseteq$. Let $x, y \in X, x \supseteq y$, be two faces with $\operatorname{dim}(y)=$ $\operatorname{dim}(x)-1$. Let $Y$ be a subset of $y^{\uparrow}$ containing $y$. Then, $x^{\uparrow \star} \backslash Y$ is contractible.

Proof We set $m=\operatorname{dim}(x)$ and $X_{0}=x^{\uparrow \star} \backslash Y$. If $m=1$, Proposition 58 is trivial ( $X_{0}$ is a singleton). Suppose now that $m \geq 2$. We denote by $y^{\prime}$ the face opposite to $y$ in $x^{\uparrow}: x=y \sqcup y^{\prime}$. Observe that $\operatorname{dim}\left(y^{\prime}\right)=0$ if $X$ is a simplicial complex and $\operatorname{dim}\left(y^{\prime}\right)=m-1$ if $X$ is a cubical complex. We will shrink $X_{0}$ to $\left\{y^{\prime}\right\}$ by removing unipolar points from $X_{0}$. First, we remove the faces of $X_{0}$ that are in $y^{\uparrow \star}$, in decreasing order relatively to their dimension. For any $(m-2)$-face $z$ in $y^{\uparrow} \backslash Y$ we derive from Lemma 57 that there are two $(m-1)$-faces in $x^{\uparrow}$ including $z$, one of which is $y$. Hence, $z$ is down unipolar in $X_{0}$ and, thanks to Properties 6 and 11

${ }^{10}$ We write $\sqcup$ for the disjoint union.

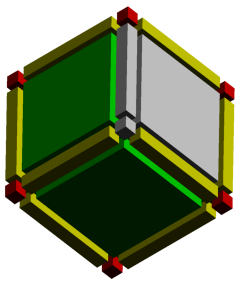

(a)

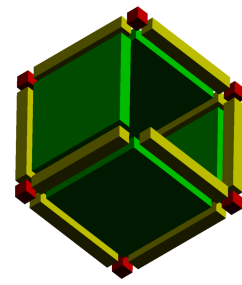

(b)

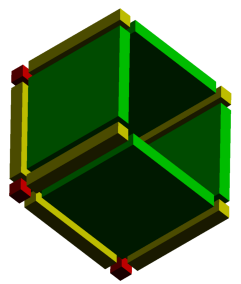

(c)

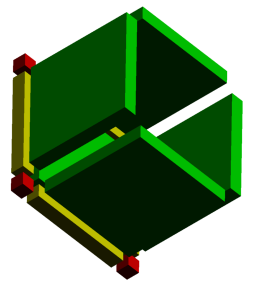

(d)

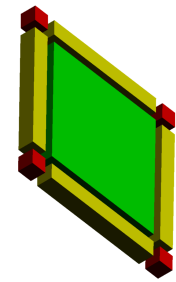

(e)
Fig. 27 Some steps of the proof of Proposition 58 (a) The set $x^{\uparrow \star}$ with $\operatorname{dim}(x)=3$. In grey, the subset $Y$ ( $y$ is the 2-face in $Y$ ). (b) The set $X_{0}=x^{\uparrow \star} \backslash Y$. (c) The set $Z$. (d) The set $Z \backslash\left\{z \notin y^{\uparrow \uparrow} \mid \operatorname{dim}(z)=1\right\}$. (e) The cell $y^{\prime \uparrow}$.

we deduce that the set $X_{1}=\left\{z \in X_{0} \mid z \notin y^{\uparrow}\right.$ or $\left.\operatorname{dim}(z)<m-2\right\}$ is a strong deformation retract of $X_{0}$. Since, according to Lemma 57 any $(m-k)$-face in $y^{\uparrow \star}$ is covered by exactly $k$ faces in $x^{\uparrow}$ and by $k-1$ faces in $y^{\uparrow}$, we can inductively remove all faces of $y^{\uparrow}$ from $X_{0}$ with the same argumentation as above. Hence, $Z=X_{0} \backslash y^{\uparrow}$ is a strong deformation retract of $X_{0}$. In a second step, we are going to prove that the faces in $Z \backslash y^{\prime \uparrow}$ are successively up unipolar if we remove them in an increasing order with respect to their dimension. Note that, since $x=y \sqcup y^{\prime}$, there is no 0 -face in $Z \backslash y^{\prime \uparrow}$. So, let us suppose that we have removed all faces in $Z \backslash y^{\uparrow}$ of dimension less than $k(1 \leq k \leq m-1)$ and let $z$ be a $k$-face in $Z \backslash y^{\prime \uparrow}$. If $X$ is a cubical complex, Lemma 57-(ii) ensures that there exists in $z^{\uparrow \star}$ exactly one $(k-1)$-face in $y^{\prime \uparrow}$, namely $z \cap y^{\prime}$, and, if $X$ is a simplicial complex, obviously $z^{\uparrow \star} \cap y^{\uparrow}=\left\{y^{\prime}\right\}$. Thus, $z$ is up unipolar in $Z_{k}=\{t \in Z \mid \operatorname{dim}(t) \geq k\} \cup y^{\uparrow}$. So, we can inductively prove that $y^{\prime \uparrow}$ is a strong deformation retract of $X_{0}$. As any cell is contractible (Property 3), we are done.

\section{Proof of Theorem 27}

Lemma 59 Let $X$ be a cubical or a simplicial complex. Let $0 \leq k<$ $m \leq n, x \in X$ be an $m$-face and $y \in x^{\uparrow}$ be a $k$-face. The set $x^{\uparrow} \cap y^{\downarrow \star}$ is homeomorphic to an abstract simplicial $(m-k-1)$-cell with an homeomorphism $\varphi$ such that $\operatorname{dim}(\varphi(z))=\operatorname{dim}(z)-k-1$ for all $z \in$ $x^{\uparrow} \cap y^{\downarrow \star}$.

Proof Let $V$ be the set of $(k+1)$-faces in $x^{\uparrow} \cap y^{\downarrow \star}$. By Lemma 57 we have $\operatorname{Card}(V)=m-k$. We will prove by induction that $x^{\uparrow} \cap y^{\downarrow \star}$ is homeomorphic to $2^{V}$ (equipped with the inclusion). Let $i$ be an integer such that $k \leq i<m$ and $x_{i}$ be an $i$-face including $y$ and included in $x$. Let $V_{i}$ be the set of $(k+1)$-faces in $x_{i}^{\uparrow} \cap y^{\downarrow \star}\left(V_{i}\right.$ is a subset of $\left.V\right)$. Suppose that $V_{i}=\emptyset$ or we have defined an homeomorphism $\varphi_{i}: x_{i}^{\uparrow} \cap y^{\downarrow \star} \rightarrow$ $2^{V_{i}} \backslash\{\emptyset\}$ such that $\operatorname{Card}(\varphi(z))=\operatorname{dim}(z)-k-1$ for all $z \in x_{i}^{\uparrow} \cap y^{\downarrow \star}$. Let $x_{i+1}$ be a face including $x_{i}$ and included in $x$ and $V_{i+1}$ be the set of $k+1$-faces in $x_{i+1}^{\uparrow} \cap y^{\downarrow \star}$. From Lemma 57 $(i)$, we deduce that there exists a face $a$ in $V$ such that $V_{i+1}=V_{i} \sqcup\{a\}$. Let $x_{i}^{\prime}$ be the face included in $x_{i+1}$ such that 
$x_{i+1}=x_{i} \sqcup x_{i}^{\prime}\left(\operatorname{dim}\left(x_{i}^{\prime}\right)=0\right.$ if $X$ is a simplicial complex and $\operatorname{dim}\left(x_{i}^{\prime}\right)=i$ if $X$ is a cubical complex). Let $Y$ be the set of faces in $x^{\uparrow}$ that intersect both $x_{i}$ and $x_{i}^{\prime}$. We define the function $\theta: Y \rightarrow x_{i}^{\uparrow}$ by $\theta(z)=z \cap x_{i}$. By Lemma 57 (ii), $\theta$ is a bijection. Let $b$ be a face in $x_{i+1}^{\uparrow} \cap y^{\downarrow \star}$. If $b \in x_{i}^{\uparrow}$, we set $\varphi_{i+1}(b)=\varphi_{i}(b)$. If $b \notin x_{i}^{\uparrow}$, then $b$ intersects both $x_{i}$ and $x_{i}^{\prime}\left(b \cap x_{i}\right.$ is not empty for it includes $y$ ) so we can set $\varphi_{i+1}(b)=\{a\} \cup \varphi_{i}(\theta(b))$. Conversely, for all $Z \in 2^{V_{i+1}} \backslash\{\emptyset\}$, we set $\psi(Z)=\varphi_{i}^{-1}(Z)$ if $a \notin Z$, $\psi(Z)=\theta^{-1}\left(\varphi_{i}^{-1}(Z \backslash\{a\})\right)$ otherwise. It is plain that $\varphi_{i+1}$ is a bijection whose inverse is $\psi$. Let $b$ be face in $x_{i+1}^{\uparrow} \cap y^{\downarrow \star}$. If $b \in x_{i}^{\uparrow}, \operatorname{dim}\left(\varphi_{i+1}(b)\right)=$ $\operatorname{dim}\left(\varphi_{i}(b)\right)=\operatorname{dim}(b)-k-1$ by the induction hypothesis. If $b \notin x_{i}^{\uparrow}$, $\operatorname{dim}\left(\varphi_{i+1}(b)\right)=1+\operatorname{dim}\left(\varphi_{i}(\theta(b))=1+\operatorname{dim}(\theta(b))-k-1=\operatorname{dim}(b)-k-1\right.$ (for the last equality, we use Lemma $57\left(\right.$ ii)). Since $2^{V_{i+1}} \backslash\{\emptyset\}$ is a cell, from the equality $\operatorname{dim}\left(\varphi_{i+1}(b)\right)=\operatorname{dim}(b)-k-1$, we infer that $\varphi_{i+1}$ is non-decreasing. As $\varphi_{i+1}$ is a bijection, $\varphi_{i+1}^{-1}$ is also non-decreasing and, thanks to Property $2 \varphi_{i+1}$ is an homeomorphism.

Proposition 58 could be stated in terms of $\beta$-simplicity: let $x<y$ be two points in a complex $X$ equipped with the order $\supseteq$ and $Y$ be a set such that $y \in Y \subseteq y^{\uparrow}$. Then, the point $x$ is a $\beta$-simple point in $X \backslash Y$. It is no longer true if we remove the condition $\operatorname{dim}(y)=\operatorname{dim}(x)-1$ (see 25] for counterexamples). Nevertheless, it remains true if we replace $\beta$-simplicity by $\gamma$-simplicity.

Proposition 60 Let $X$ be a cubical or a simplicial complex equipped with the order $\supseteq$. Let $x$ be a face in $X$ and $Y \subset x^{\uparrow \star}$ be a subset that has a maximum or a minimum. Then, the point $x$ is $\gamma$-simple in $X \backslash Y$.

Proof Proving that $x$ is $\gamma$-simple in $X \backslash Y$ comes down to establish that $x^{\uparrow \star} \backslash Y$ is homotopically trivial. First case: $Y$ has a minimum noted $y$. The proof is made by induction on $m$, the dimension of $x$. If $m=1$, the result is obvious. We assume now that $m \geq 2$. If $\operatorname{dim}(y)=m-1$, we apply Proposition 58 If $\operatorname{dim}(y) \leq m-2$, let $z$ be an $(m-1)$-face of $x^{\uparrow}$ including $y$. From the induction hypothesis, $z^{\uparrow \star} \backslash Y$ is homotopically trivial. Hence, $z$ is a $\gamma$-point in $x^{\uparrow \star} \backslash Y$. So, $x^{\uparrow \star} \backslash(Y \cup\{z\})$ is weakhomotopy equivalent to $x^{\uparrow \star} \backslash Y$ (Property 22]. Now, from Proposition 58. we deduce that $x^{\uparrow \star} \backslash(Y \cup\{z\})$ is contractible and we are done: by transitivity, $x^{\uparrow \star} \backslash Y$ is homotopically trivial.

Second case: $Y$ has a maximum noted $y$. The proof is made by induction on $\operatorname{Card}(Y)$. If $\operatorname{Card}(Y)=1$, i.e., $Y=\{y\}$, we use the first part of the proof to conclude. Suppose now that $\operatorname{Card}(Y) \geq 2$. Let $z, z \neq y$, be a face in $Y$ such that $\operatorname{dim}(z)=\min \{\operatorname{dim}(t) \mid t \in Y \backslash\{y\}\}$. We observe that $z^{\uparrow \star} \cap Y=\{y\}$. Now, we set $Z=\left(x^{\uparrow \star} \backslash Y\right) \cup\{z\}=x^{\uparrow \star} \backslash(Y \backslash\{z\})$. As $\operatorname{Card}(Y \backslash\{z\})<\operatorname{Card}(Y)$, we deduce from the induction hypothesis that $Z$ is homotopically trivial. Let us prove that $z$ is a $\gamma$-point for $Z$. We have $z^{\uparrow \star} \cap Z=z^{\uparrow \star} \backslash\{y\}$ which, from the first part of the proof, is homotopically trivial. Hence, $z$ is a $\gamma$-simple point for $Z$. Thereafter, the injection $i: x^{\uparrow \star} \backslash Y \rightarrow Z$ is a weak homotopy equivalence and we conclude straightforwardly.

\section{Proof (Theorem 27)}

Let $y \in Y \subset X$ be a $\beta$-simple point in $Y$. Then $y^{\uparrow \star} \cap Y$ or $y^{\downarrow \star} \cap Y$ is contractible. We suppose first that $y^{\uparrow \star} \cap Y$ is contractible. From Corollary 9 we know that there exists a sequence $\left(x_{i}\right)_{i=0}^{r}(r \geq 0)$ such that $y^{\uparrow \star} \cap Y=\left\{x_{i}\right\}_{i=0}^{r}$ and $x_{j}$ is unipolar in $\left\{x_{i}\right\}_{i=0}^{j}$ for all $j \in[1, r]$. The proof consists to establish that $x_{j}$ is a $\gamma$-simple point in $y^{\uparrow \star} \backslash\left\{x_{i}\right\}_{i=0}^{j-1}$ for all $j \in[1, r]$. This will imply (by transitivity) that the injection of $y^{\uparrow \star} \backslash Y$ in $y^{\uparrow \star} \backslash\left\{x_{0}\right\}$ is a weak homotopy equivalence. Then Proposition 60 will permit us to conclude easily. So, let us suppose first that $x_{j}$ is up-unipolar in $\left\{x_{i}\right\}_{i=0}^{j}$ for some $j \in[1, r]$. We set $Y_{j}=x_{j}^{\uparrow \star} \cap\left\{x_{i}\right\}_{i=0}^{j}$. From Proposition 60 we derive that $x_{j}^{\uparrow \star} \backslash Y_{j}$ is homotopically trivial (since $Y_{j}$ has a minimum). As $x_{j}^{\uparrow \star} \cap\left(y^{\uparrow \star} \backslash\left\{x_{i}\right\}_{i=0}^{j-1}\right)=x_{j}^{\uparrow \star} \backslash Y_{j}, x_{j}$ is a $\gamma$-simple point in $y^{\uparrow \star} \backslash\left\{x_{i}\right\}_{i=0}^{j-1}$. We suppose now that $x_{j}$ is downunipolar in $\left\{x_{i}\right\}_{i=0}^{j}$ and we set $Y_{j}=x_{j}^{\downarrow \star} \cap\left\{x_{i}\right\}_{i=0}^{j}$. We observe that $Y_{j}$ has a maximum. Thanks to Lemma 59 we can consider an homeomorphism $\varphi: y^{\uparrow} \cap x_{j}^{\downarrow \star} \rightarrow Z$ where $Z$ is a simplicial cell. From Property 2 (any continuous function between posets is non-decreasing), we derive that $\varphi\left(\left(y^{\uparrow \star} \cap x_{j}^{\downarrow \star}\right)\right)=\varphi(y)^{\uparrow \star}$ and that $\varphi\left(Y_{j}\right)$ has a maximum (for $Y_{j}$ has a maximum). Then we invoke Proposition 60 to assert that $\varphi\left(\left(y^{\uparrow \star} \cap x_{j}^{\downarrow \star}\right) \backslash Y_{j}\right)=\varphi(y)^{\uparrow \star} \backslash \varphi\left(Y_{j}\right)$ is homotopically trivial. Hence, $\left(y^{\uparrow \star} \cap x_{j}^{\downarrow \star}\right) \backslash Y_{j}=x_{j}^{\downarrow \star} \cap\left(y^{\uparrow \star} \backslash\left\{x_{i}\right\}_{i=0}^{j-1}\right)$ is homotopically trivial and $x_{j}$ is a $\gamma$-point in $y^{\uparrow \star} \backslash\left\{x_{i}\right\}_{i=0}^{j-1}$.

We suppose now that $y^{\downarrow \star} \cap Y$ is contractible. Taking the reverse order on $X$ (since $X=\mathbb{F}^{n},(X, \leq)$ is homeomorphic to $(X, \geq)$ ), we derive from Proposition 14 that $y$ is a $\beta$-simple point for $Y$ and from Corollary 10 that $y^{\uparrow \star} \cap Y$ is contractible. Then it follows from the first part of the proof that $y$ is a $\gamma$-simple point for $(X \backslash Y) \cup\{y\}$ equipped with the inclusion and we conclude, invoking Proposition 20 that $y$ is a $\gamma$ simple point for $(X \backslash Y) \cup\{y\}$ with the initial order.

\section{Counterexamples}

Counterexample 61 (Theorem 27) Figure 28 illustrates the fact that Theorem 27 is generally false when the poset $(X, \leq)$ is a cubical complex, but $(X, \geq)$ is not a cubical complex.

Counterexample 62 (Proposition 32) Figure 29 illustrates the fact that Proposition 32 is generally false in a non-distributive lattice.

Counterexample 63 (Proposition 44) Figure 30 illustrates the fact that Proposition 44 is generally false in a non-distributive lattice. Furthermore, this figure shows that the number of connected components of the supports is not preserved by a cut in such a lattice. Therefore, this counterexample is also a counterexample for Theorem 47 when the lattice is not distributive.

Counterexample 64 (Theorem 47) Figure 31 illustrates the fact that Theorem 47 is generally false if the poset $X$ has not the pierced sphere property.

Counterexample 65 (Proposition 50) Figure 32 shows that if the lattice $T$ is not distributive, the binary image $\mu \wedge$ a where $\mu$ is a regular image and $a$ is an atom of $T$ can be non-regular.

Counterexample 66 (Proposition 56 Figure 33 shows that in Proposition 56. Condition (iii) is not necessary.

\section{E Comparison between ML-simple points and digitally simple points}

In Figure 34, we borrow the images used in [15] to illustrate the notion of ML-simple point in label digital images in order to compare this notion with our own notion of digitally simple point in regular label images (we have omitted the first image of [15] which is very similar to the second one).

\section{References}

1. O. P. Buneman, A grammar for the topological analysis of plane figures, in: B. Meltzer, D. Michie (Eds.), Machine Intelligence, Vol. 5, 1969, pp. 383-393. 1

2. T. Y. Kong, A digital fundamental group, Computers \& Graphics 13 (2) (1989) 159-166. 1, 3 


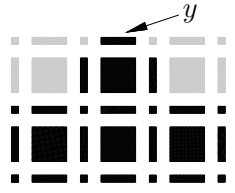

(a)

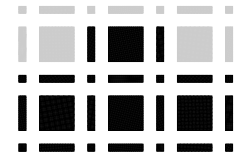

(b)
Fig. 28 (a) A set $X$ which is a cubical complex but whose dual is not a complex (because of the boundary). In black, a subset $Y$ of $X$. The point $y$ is a 1-face of $Y$. In light grey, the complement of $Y$ in $X, X \backslash Y$. (b) In black, the set $Y \backslash\{y\}$. In light grey, the set $(X \backslash Y) \cup\{y\}$. Clearly, $y$ is a $\beta$-simple for $Y$ ( $y$ is up-unipolar in $Y$ ) but $y$ is not $\gamma$-simple for $(X \backslash Y) \cup\{y\}$ since this later set has not the same number of connected components as $X \backslash Y$.

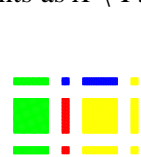

(a)

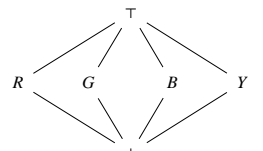

(b)

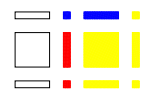

(c)
Fig. 29 (a) A label image $\mu: X \rightarrow T$. (b) The Hasse diagram of $T$ (which is not distributive). The labels $\perp, R, G, B, Y, \top$ are depicted respectively in white, red, green, blue, yellow and black. The yellow 2face $x$ is not simple for the label T since the label $G$ is such that $G \wedge Y=$ $\perp$ and $G \wedge \top \neq \perp$ but $x$ is not $\beta$-simple for $\langle G\rangle \cup\{x\}$. (c) The label image $\varphi \circ \mu: X \rightarrow \varphi(T)$ (for the definition of $\varphi$, see Proposition 32). In this image, the point $x$ is simple for the label $\mathrm{T}$.

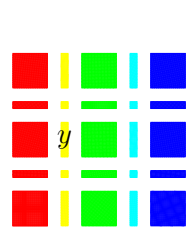

(a)

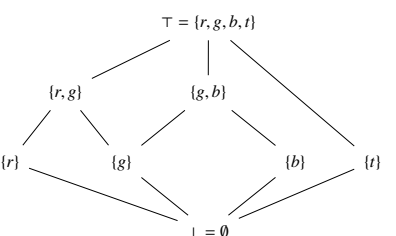

(b)

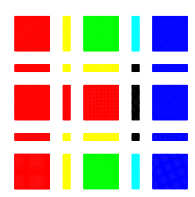

(c)
Fig. 30 (a) A closed supports label image $\mu: X \rightarrow$ $T$ with $T=\{\emptyset,\{r\},\{g\},\{b\},\{t\},\{r, g\},\{g, b\},\{r, g, b, t\}\}$, equipped with the inclusion. (b) The Hasse diagram of $T$. The labels $\{r\},\{g\},\{b\},\{r, g\},\{g, b\},\{r, g, b, t\}$ are depicted respectively in red, green, blue, yellow, cyan and black. (c) The cut $\mu_{y,\{g\}}$. In the image $\mu$, the support of $t$ is empty. But, in the cut $\mu_{y, g}$, the support of $t$ is no longer empty (it contains the three points in black).

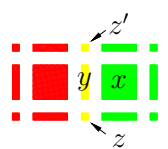

(a)

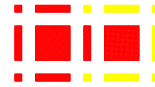

(b)
Fig. 31 (a) An image $\mu: X \rightarrow 2^{\{r, g\}}$. In the poset $X$, the points $z$ and $z^{\prime}$ are identified. Thus, $X$ has not the pierced sphere property $\left(x^{\uparrow \star} \backslash\{y\}\right.$ is a ring). The support of $\{g\}$ is a ball. (b) The cut $\mu_{y,\{g\}}$. The support of $\{g\}$ is a ring.

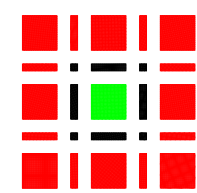

(a)

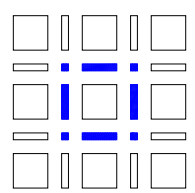

(b)
Fig. 32 (a) A regular image $\mu: X \rightarrow T$ with $T=$ $\{\emptyset,\{r\},\{g\},\{b\},\{r, g, b\}\}$ equipped with the inclusion. (b) The binary image $\mu \wedge\{b\}$ which is not regular.

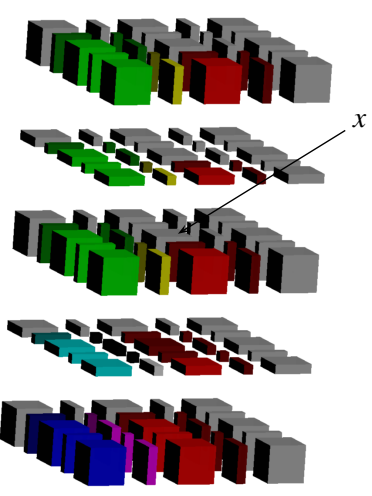

(a)

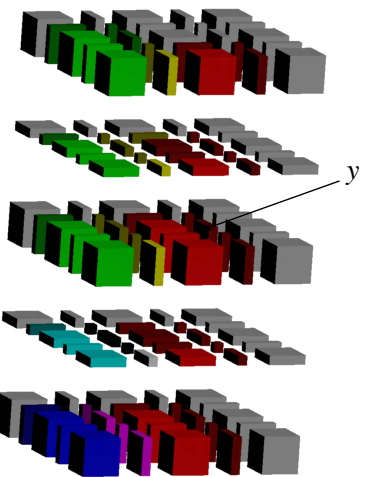

(c)

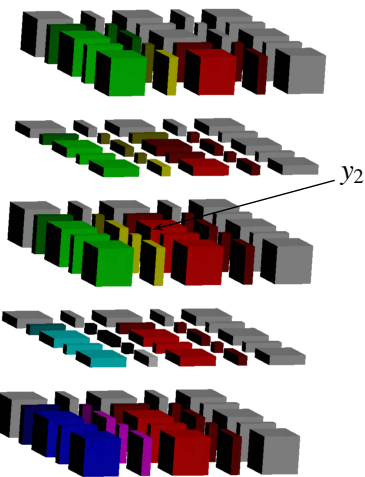

(e)
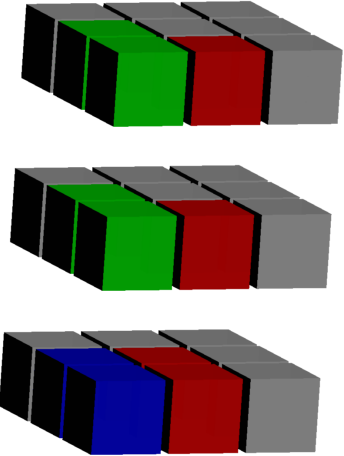

(b)

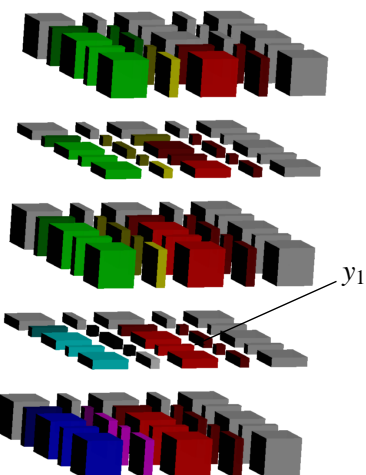

(d)

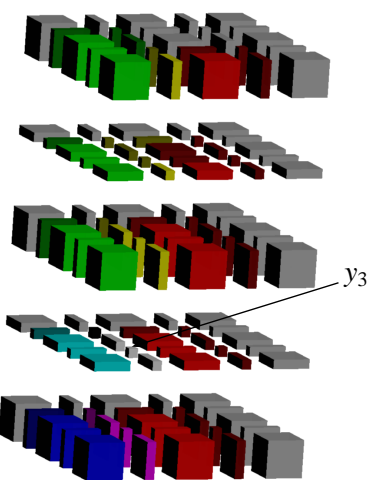

(f)
Fig. 33 (a) A regular image $\mu: X \rightarrow 2^{\{r, g, b, e\}}$ where the four protolabels $r, g, b, e$ are depicted respectively in red, green, blue and grey. We take the notations of the proof of Proposition 56 The xel $x$ is at the center of the image. Its label is $t_{0}=\{e\}$. (b) The label digital image associated to $\mu$ (in $\mathbb{Z}^{3}$ ). (c) The cut $\mu_{1}=\mu_{y, t_{0}}$. (d) The cut $\mu_{2}=\left(\mu_{1}\right)_{y_{1}, t_{0}}$. (e) The cut $\mu_{3}=\left(\mu_{2}\right)_{y_{2}, t_{0}}$. (f) The cut $\mu_{4}=\left(\mu_{3}\right)_{y_{3}, t_{0}}$ which is regular. Hence, the xel $x$ is digitally simple. Nevertheless we have $\mu\left(y_{3}\right)=\bigvee\{r, g, b, e\}$, so Condition (iii) of Proposition 56 is not satisfied. 
1

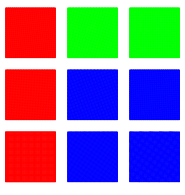

(a)

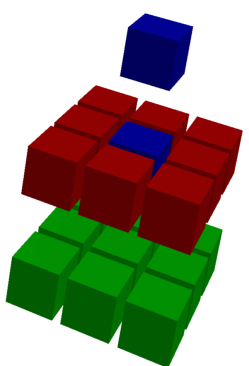

(e)

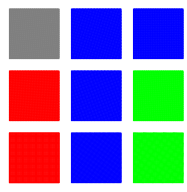

(b)

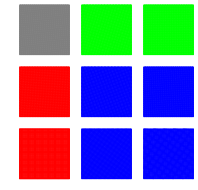

(c)

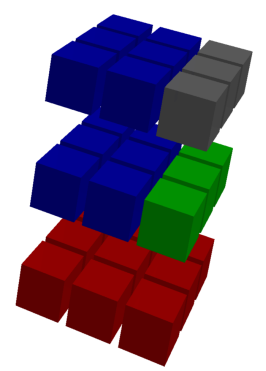

(f)

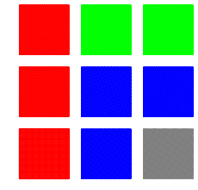

(d)

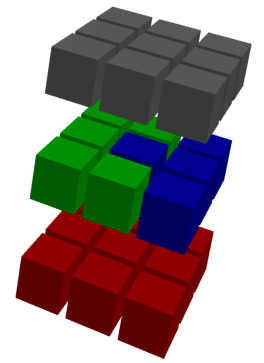

(g)
Fig. 34 These seven label images must be considered as defined on $\mathbb{Z}^{n}$ for ML-simple points or on $\mathbb{F}^{n}$ for digitally simple points. In the latter case, we assume the image to be regular, so there is no need to represent the faces of dimension less than or equal to $n-1$. There are four proto-labels depicted in red, green, blue and grey. The voxel $x$ is the central voxel (in blue). The test consists in checking if the voxel $x$ is (ML or $\mathrm{x}$ )-simple for the red label. Note that ML-simple points are to be used with the $(4,8)$ or the $(6,18)$ adjacency pair. (a) $x$ is MLsimple [15] and it can easily be seen that $x$ is digitally simple. (b) to (g) $x$ is not ML-simple [15] and $x$ is not digitally simple (these checks are not difficult and left to the reader). Observe that on subfigure (f), the grey label is not taken into account to decide that $x$ is not a ML-simple point. Likely, the authors of [15] have chosen to add a fourth label here to put in evidence that the move of the central voxel from the blue label to the red label could modify the topology of the green label. It is different with digitally simple points. Because of the grey label, neither Condition $i$ nor Condition iii of Proposition 56 are satisfied. But, if we replace the grey label by the green label, $x$ becomes digitally simple.

3. R. González-Díaz, P. Real, On the cohomology of 3D digital images, Discrete Applied Mathematics 147 (2005) 245-263, advances in Discrete Geometry and Topology. 1

4. C. J. Hilditch, Linear skeletons from square cupboards., in: B. Meltzer, D. Michie (Eds.), Machine Intelligence, Vol. 4, 1969, pp. 403-420. 1

5. G. Bertrand, On P-simple points, Comptes Rendus de l'Académie des Sciences, Série Mathématiques 1 (321) (1995) 1077-1084. 1

6. C. Ronse, A topological characterization of thinning, Theoretical Computer Science 43 (0) (1986) 31-41. 1

7. N. Passat, L. Mazo, An introduction to simple sets, Pattern Recognition Letters 30 (15) (2009) 1366-1377. 1

8. J.-F. Mangin, V. Frouin, I. Bloch, J. Régis, J. López-Krahe, From $3 \mathrm{D}$ magnetic resonance images to structural representations of the cortex topography using topology preserving deformations, Journal of Mathematical Imaging and Vision 5 (4) (1995) 297-318. 1 .

9. S. Miri, N. Passat, J.-P. Armspach, Topology-preserving discrete deformable model: Application to multi-segmentation of brain MRI, in: International Conference on Image and Signal Processing - ICISP 2008, Vol. 5099 of Lecture Notes in Computer Science, Springer, 2008, pp. 67-75. 1

10. F. Poupon, J.-F. Mangin, D. Hasboun, C. Poupon, I. Magnin, V. Frouin, Multi-object deformable templates dedicated to the segmentation of brain deep structures, in: MICCAI'98: Proceedings of the First International Conference on Medical Image Computing and Computer-Assisted Intervention, Springer-Verlag, 1998, pp. 1134-1143. 1]

11. P.-L. Bazin, D. Pham, Topology-preserving tissue classification of magnetic resonance brain images, IEEE Transactions on Medical Imaging 26 (4) (2007) 487-496. 1

12. J. Liu, S. Huang, W. Nowinski, Registration of brain atlas to MR images using topology preserving front propagation, Journal of Signal Processing Systems 55 (1) (2009) 209-216. 1.

13. A. Rosenfeld, Connectivity in digital pictures, Journal of the Association for Computer Machinery 17 (1) (1970) 146-160. 2

14. O. Duda, P. E. Hart, J. H. Munson, Graphical data processing research study and experimental investigation, Tech. Rep. AD650926, Stanford Research Institute (1967). 2

15. G. Damiand, A. Dupas, J.-O. Lachaud, Fully deformable 3D digital partition model with topological control, Pattern Recognition Letters 32 (2011) 1374-1383. 22, 22, 26

16. G. Bertrand, Simple points, topological numbers and geodesic neighborhoods in cubic grids, Pattern Recognition Letters 15 (1994) 1003-1011. 2

17. L. J. Latecki, Multicolor well-composed pictures, Pattern Recognition Letters 16 (4) (1995) 425-431. 2

18. S. Siqueira, L. Latecki, N. Tustison, J. Gallier, J. Gee, Topological repairing of 3D digital images, Journal of Mathematical Imaging and Vision 30 (3) (2008) 249-274. 2

19. Y. Cointepas, I. Bloch, L. Garnero, A cellular model for multi-objects multi-dimensional homotopic deformations, Pattern Recognition 34 (2001) 1785-1798. 2]

20. P.-L. Bazin, L. Ellingsen, D. Pham, Digital homeomorphisms in deformable registration, in: N. Karssemeijer, B. Lelieveldt (Eds.), IPMI, Vol. 4584 of Lecture Notes in Computer Science, Springer, 2007, pp. 211-222. 22, 32

21. J. H. C. Whitehead, Simplicial Spaces, Nuclei and m-Groups, Proceedings of the London Mathematical Society s2-45 (1939) 243327. 2, 5,14

22. L. Mazo, N. Passat, M. Couprie, C. Ronse, Digital imaging: A unified topological framework, Journal of Mathematical Imaging and Vision (doi: 10.1007/s10851-011-0308-9). 3, 10, 11,22

23. G. Bertrand, M. Couprie, Two-dimensional thinning algorithms based on critical kernels, Journal of Mathematical Imaging and Vision 31 (1) (2008) 35-56. 520

24. J. Munkres, Topology, Prentice Hall, 1999.

25. L. Mazo, N. Passat, M. Couprie, C. Ronse, Paths, homotopy and reduction in digital images, Acta Applicandae Mathematicae 113 (2) (2011) 167-193. 6, 7, 19,24

26. R. Bing, Some aspects of the topology of 3-manifolds related to the Poincaré conjecture, Lectures on Modern Mathematics II (1964) 93-128. 5

27. E. Zeeman, On the dunce hat, Topology 2 (1964) 341-358. 5

28. J. H. C. Whitehead, Combinatorial homotopy. I., Bulletin of the american Mathematical Society 55 (1949) 213-245. 4

29. S. Fourey, R. Malgouyres, A concise characterization of 3D simple points, Discrete Applied Mathemathics 125 (1) (2003) 59-80. 4,10

30. C. R. F. Maunder, Algebraic Topology, Dover, 1996. 4

31. J. Munkres, Elements of Algebraic Topology, Westview Press, 1996. 4

32. A. May, A Concise Course in Algebraic Topology, U. Chicago Press, 1999. 4

33. A. Hatcher, Algebraic Topology, Cambridge University Press, 2002. 4

34. P. Giblin, Graphs, surfaces and homology, Cambridge University Press, 2010. 4, 5

35. P. Alexandroff, Diskrete Räume, Rec. Math. [Mat. Sbornik] N.S. (1937) 501-519. 5, 60

36. G. Birkhoff, Rings of sets, Duke Mathematical Journal 3 (3) (1937) 443-454. 5 
37. M. McCord, Singular homology groups and homotopy groups of finite topological spaces, Duke Mathematical Journal 33 (3) (1966) 465-474. 607

38. G. Bertrand, New notions for discrete topology, in: DCGI '99: Proceedings of the 8th International Conference on Discrete Geometry for Computer Imagery, 1999, pp. 218-228. 6, 80

39. R. E. Stong, Finite topological spaces, Transactions of the American Mathematical Society 123 (25) (1966) 325-340. 6, 7

40. J. P. May, Finite topological spaces (lecture notes), url : www.math.uchicago.edu/ may/MISC/FiniteSpaces.pdf (2008). 6, 7

41. J. A. Barmak, E. G. Minian, Simple homotopy types and finite spaces, Advances in Mathematics 218 (2008) 87-104. 60

42. J. A. Barmak, E. G. Minian, One-point reductions of finite spaces, h-regular CW-complexes and collapsibility, Algebraic \& Geometric Topology 8 (3) (2008) 1763-1780. 9

43. T. Y. Kong, A. Rosenfeld, Digital topology: Introduction and survey, Computer Vision, Graphics, and Image Processing 48 (1989) 357-393. 10

44. V. A. Kovalevsky, Finite topology as applied to image analysis, Computer Vision, Graphics, and Image Processing 46 (2) (1989) 141-161. 10, 11

45. V. Kovalevsky, Axiomatic digital topology, Jounal of Mathematical Imaging and Vision 26 (1) (2006) 41-58. 10

46. V. Kovalesky, Geometry of Locally Finite Spaces, Publishing House Dr. Baerbel Kovalevski, 2008. 10

47. G. T. Herman, Geometry of Digital Spaces, Birkhuser, 1998. 10

48. E. Kronheimer, The topology of digital images, Topology and its Applications 46 (1992) 279-303. 10

49. C. Ronse, V. Agnus, Morphology on label images: Flat-type operators and connections, Journal of Mathematical Imaging and Vision 22 (2) (2005) 283-307. 11

50. C. Ronse, V. Agnus, Geodesy on label images, and applications to video sequence processing, Journal of Visual Communication and Image Representation 19 (2008) 392-408. 11

51. R. Ayala, E. Domínguez, A. Francés, A. Quintero, Digital lighting functions, in: procs. Discrete Geometry for Computer Imagery, LNCS, Springer Verlag, Vol. 1347, 1997, pp. 139-150. 11

52. C. Ronse, An isomorphism for digital images, Journal of Combinatorial Theory, Series A 39 (2). 13

53. E. H. Spanier, Algebraic Topology, Springer, 1994. 16

54. T. Y. Kong, Topology-preserving deletion of 1's from 2-, 3- and 4-dimensional binary images, in: DGCI '97: Proceedings of the 7th International Workshop on Discrete Geometry for Computer Imagery, Springer-Verlag, 1997, pp. 3-18. 20

55. M. Couprie, G. Bertrand, New characterizations of simple points in 2D, 3D and 4D discrete spaces, IEEE Transactions on Pattern Analysis and Machine Intelligence 31 (4) (2009) 637-648. 21

56. T. Blyth, Lattices and Ordered Algebraic Structures, Springer, London, 2005. 22

57. G. Grätzer, General Lattice Theory, 2nd Edition, Birkhäuser, Basel, 2003. 22 